\title{
NEW NUMBER FIELDS WITH KNOWN $p$-CLASS TOWER
}

\author{
DANiel C. MAYer \\ Respectfully dedicated to Professor Igor R. Shafarevich
}

\begin{abstract}
The $p$-class tower $\mathrm{F}_{p}^{\infty}(k)$ of a number field $k$ is its maximal unramified pro- $p$ extension. It is considered to be known when the $p$-tower group, that is the Galois group $G:=\operatorname{Gal}\left(\mathrm{F}_{p}^{\infty}(k) \mid k\right)$, can be identified by an explicit presentation. The main intention of this article is to characterize assigned finite 3 -groups uniquely by abelian quotient invariants of subgroups of finite index, and to provide evidence of actual realizations of these groups by 3-tower groups $G$ of real quadratic fields $K=\mathbb{Q}(\sqrt{d})$ with 3-capitulation type (0122) or (2034).
\end{abstract}

\section{Introduction}

Given a prime $p$, the Hilbert $p$-class field tower $\mathrm{F}_{p}^{\infty}(k)$ is the maximal unramified pro- $p$ extension of an algebraic number field $k$. It is considered to be known if a presentation of its Galois group is given (and not merely its length $\ell_{p}(k)$ ). The key for determining the Galois group

$$
G:=\mathrm{G}_{p}^{\infty}(k)=\operatorname{Gal}\left(\mathrm{F}_{p}^{\infty}(k) \mid k\right),
$$

which is briefly called the $p$-class tower group of $k$, is the structure of $p$-class groups $\mathrm{Cl}_{p}(L)$ of unramified (abelian or non-abelian) $p$-extensions $L \mid k$, collected in IPADs (index-p abelianization data) 21. Our main goal is to present new criteria for the occurrence of assigned 3-class tower groups $G$ in terms of their IPADs and proofs of their actual realization by suitable base fields $k$.

Complex quadratic fields $k=\mathbb{Q}(\sqrt{d})$ with negative fundamental discriminant $d<0$ were the first objects whose $p$-class tower has been studied for an odd

(C) 2015 Mathematical Institute, Slovak Academy of Sciences.

2010 Mathematics Subject Classification: Primary 11R37, 11R29, 11R11, 11R16, 11R20; Secondary 20D15, 20F05, 20F12, 20F14, 20-04.

Keywords: p-class field towers, p-capitulation, $p$-class groups, quadratic fields, cubic fields, dihedral fields; finite $p$-groups, descendant trees, $p$-group generation algorithm.

Research was supported by the Austrian Science Fund (FWF): P 26008-N25. 
prime $p$ by A. Scholz and O. Taussky in 1934 [27. These authors used group theory to classify the 3 -capitulation type $\varkappa_{1}(k)=\left(\operatorname{ker}\left(j_{i}\right)\right)_{1<i<4}$ of complex quadratic fields $k$ with 3 -class group $\mathrm{Cl}_{3}(k) \simeq(3,3)$ and four class extension homomorphisms $j_{i}: \mathrm{Cl}_{3}(k) \rightarrow \mathrm{Cl}_{3}\left(L_{i}\right)$ to the unramified cyclic cubic superfields $L_{i}, 1 \leq i \leq 4$, into 13 possible cases, denoting sections of similar types with upper case letters D,E,F,G,H and proving that other sections A,B,C are impossible (B,C in general, A for quadratic fields).

In the present article, we use IPADs of 2 nd order to show that real quadratic fields $K=\mathbb{Q}(\sqrt{d})$ with one of the 3 -capitulation types c.18, $\varkappa=(0122)$, and c.21, $\varkappa=(2034)$, where 0 denotes a total capitulation [17, p. 477], have 3-class field towers of exact length $\ell_{3}(K)=\mathbf{3}$. These types are strange because they are unique with the following properties: Every finite metabelian 3-group $\mathfrak{G}$ with one of these types $\varkappa_{1}(\mathfrak{G})$ has coclass $c c(\mathfrak{G})=2$ and is infinitely capable with nuclear rank $\mathrm{NR}(\mathfrak{G}) \geq 1$ [20]. So $\mathfrak{G}$ cannot be leaf of a tree. The second 3 -class group $\mathfrak{G}:=\mathrm{G}_{3}^{2}(K)=\operatorname{Gal}\left(\mathrm{F}_{3}^{2}(K) \mid K\right)[16$ ] of the real quadratic fields $K=\mathbb{Q}(\sqrt{d})$, $d>0$, under investigation is such a group.

Section c (containing types 18 and 21) was found group-theoretically by B. Nebelung [23] in 1989, 55 years after the Scholz-Taussky sections $\mathrm{D}, \ldots, \mathrm{H}$. However, for nearly 20 years, examples of fields with these types, which certainly cannot occur for complex quadratic fields, were unknown, and we were the first who discovered suitable real quadratic base fields $K=\mathbb{Q}(\sqrt{d})$, namely

$d=\mathbf{5 4 0 3 6 5}$ with type c.21 on January 01, 2008, and

$d=\mathbf{5 3 4} 8 \mathbf{2 4}$ with type c.18 on August 20, 2009 [16, Tbl. 5, p. 499].

Inspired by the 1st International Conference on Groups and Algebras in Shanghai at the end of July 2015, we suddenly had the rewarding idea and the courage to study their 3 -class tower.

\section{Main result}

TheOREM 2.1. The following real quadratic fields $K=\mathbb{Q}(\sqrt{d})$, with fundamental discriminant $d>0$, 3-class group $\mathrm{Cl}_{3}(K)$ of type $(3,3)$, and 3 -capitulation type $\varkappa_{1}(K)$ belonging to the section $c$, have a 3 -class field tower

$$
K<\mathrm{F}_{3}^{1}(K)<\mathrm{F}_{3}^{2}(K)<\mathrm{F}_{3}^{3}(K)=\mathrm{F}_{3}^{\infty}(K)
$$

of exact length $\ell_{3}(K)=3$ :

(1) all the 4461 fields of type c. $18, \varkappa_{1}(K)=(0122)$ with $d<10^{9}$, and

(2) all the 4528 fields of type c.21, $\varkappa_{1}(K)=(2034)$ with $d<10^{9}$. 


\section{Second 3-class groups as tree vertices}

Before we start with required theoretical foundations in the section $\S$, let us have a glance at the two trees, where all second 3-class groups $\mathfrak{G}$ with type $\varkappa_{1}(\mathfrak{G})$ in Nebelung's Section c are located as mainline vertices. These trees first arose in our investigation of metabelian 3 -groups $\mathfrak{G}$ with type $\varkappa_{1}(\mathfrak{G})$ in the S cholz- T a us sky Section E [19, Fig. 3.6-3.7, pp. 442-443], which are represented by terminal leaves, without exceptions. They set in with $\langle 2187,288 \ldots 290\rangle$ on the tree $\mathcal{T}^{2}(\langle 243,6\rangle)$ and with $\langle 2187,302|304| 306\rangle$ on the tree $\mathcal{T}^{2}(\langle 243,8\rangle)$. The 3-tower length $\ell_{3}(k)=3$ of complex quadratic fields $k$ with the second 3-class groups $\mathfrak{G}=\operatorname{Gal}\left(\mathrm{F}_{3}^{2}(k) \mid k\right)$ of this kind was determined successfully by M. R. Bush and ourselves [11, Cor. 4.1.1, p. 775] for the first time. We discovered that the corresponding 3-class tower groups $G=\operatorname{Gal}\left(\mathrm{F}_{3}^{\infty}(k) \mid k\right)$ with $G / G^{\prime \prime} \simeq \mathfrak{G}$ are sporadic isolated vertices (outside of coclass trees) with unbounded higher coclass $\operatorname{cc}(G) \geq 3$ but with fixed derived length $\operatorname{dl}(G)=3$, as visualized in [21, Fig. 8-9, pp. 188-189]. These results concerning the section E were generalized in [22, Fig. 3-4, pp. 754-755].

Generally, the vertices of the coclass trees in the Fig. 1]and2 2 represent isomorphism classes of finite 3-groups. Two vertices are connected by an edge $H \rightarrow G$ if $G$ is isomorphic to the last lower central quotient $H / \gamma_{c}(H)$, where $c=\operatorname{cl}(H)$ denotes the nilpotency class of $H$, and $|H|=3|G|$, that is, $\gamma_{c}(H)$ is cyclic of order 3. (See also [19, $\S 2.2$, p. 410-411] and [20, $\S 4$, p. 163-164].)

The vertices of the coclass trees in both figures are classified by using different symbols:

(1) big full discs $\bullet$ represent metabelian groups $\mathfrak{G}$ with bicyclic centre of type $(3,3)$ and defect $k(\mathfrak{G})=0$ [19, $\S 3.3 .2$, p. 429],

(2) small full discs $\bullet$ represent metabelian groups $\mathfrak{G}$ with cyclic centre of order 3 and defect $k(\mathfrak{G})=1$,

(3) small contour squares $\square$ represent non-metabelian groups $\mathfrak{H}$.

A symbol $n *$ adjacent to a vertex denotes the multiplicity of a batch of $n$ immediate descendants sharing a common parent. The groups of particular importance are labelled by a number in angles, which is the identifier in the SmallGroups library [6], [7] of MAGMA [15], where we omit the orders, which are given on the left hand scale. The transfer kernel types $\varkappa_{1}$, briefly TKT [17. Thm. 2.5, Tbl. 6-7], in the bottom rectangle concern all vertices located vertically above. The first, resp., the second, component $\tau_{1}(1)$, resp., $\tau_{1}(2)$, of the transfer target type (TTT) [21, Dfn. 3.3, p. 288] in the left rectangle concerns vertices on the same horizontal level with defect $k(\mathfrak{G})=0$. The periodicity with length 2 of branches, $\mathcal{B}(j) \simeq \mathcal{B}(j+2)$ for $j \geq 7$, sets in with branch $\mathcal{B}(7)$, having a root of order $3^{7}$. The metabelian skeletons were drawn in [23, p. 189ff], the complete trees were given in [3, Fig. 4.8, p. 76, and Fig. 6.1, p. 123]. 


\section{DANIEL C. MAYER}

Figure 1 visualizes 3-groups which form the crucial objects in the section $\S 7$
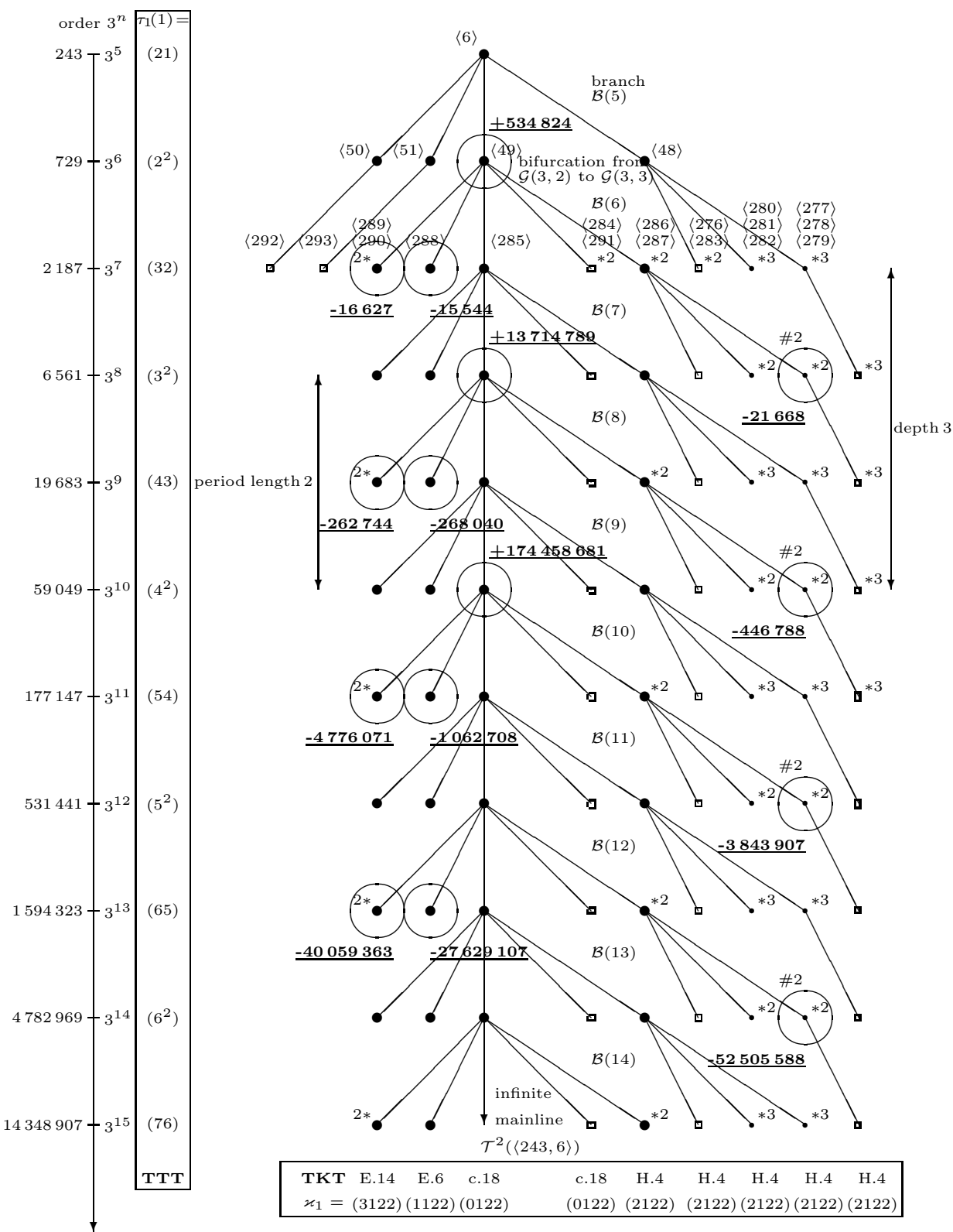
Figure 2 visualizes 3 -groups which form the crucial objects in the section $\S 8$

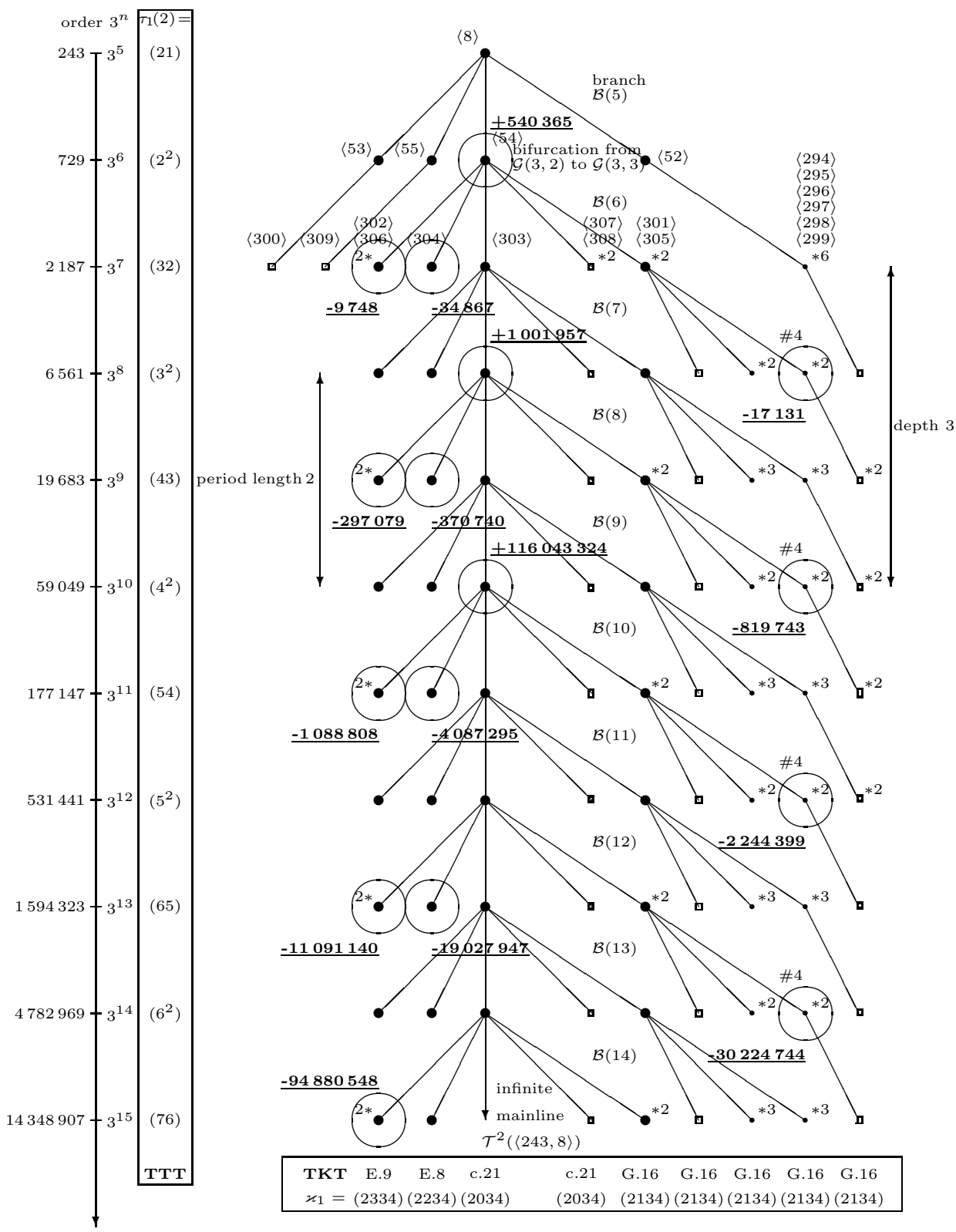

FigURE 2. 3-groups $G$ represented by vertices of the coclass tree $\mathcal{T}^{2}(\langle 243,8\rangle)$. 
The actual realization of a vertex as the second 3-class group $\mathfrak{G}=G_{3}^{2}(K)$ of a complex, resp., real, quadratic number field $K=\mathbb{Q}(\sqrt{d})$ of type $(3,3)$ with discriminant $-10^{8}<d<10^{9}$ is indicated by an underlined boldface signed discriminant (minimal in absolute value) adjacent to an oval surrounding the vertex.

The realization of mainline vertices with TKT c.18 and c.21 as $\mathfrak{G}=G_{3}^{2}(K)$, starting with $\langle 729,49\rangle$ on the tree $\mathcal{T}^{2}(\langle 243,6\rangle)$ and with $\langle 729,54\rangle$ on the tree $\mathcal{T}^{2}(\langle 243,8\rangle)$, disproved the strict leaf conjecture (that a second 3-class group must be a leaf of the metabelian skeleton of a coclass graph) but it is no violation of the weak leaf conjecture [19, Cnj. 3.1, p. 423], since these vertices do not possess metabelian immediate descendants of the same TKT with higher defect $k(\mathfrak{G})$ of commutativity.

\section{Pattern recognition via Artin transfers}

Let $p$ be a fixed prime number and $k$ be a number field with $p$-class group $\mathrm{Cl}_{p}(k)$ of order $p^{v}$, where $v \geq 0$ denotes a non-negative integer. According to the Artin reciprocity law of class field theory [1, $\mathrm{Cl}_{p}(k)$ is isomorphic to the Galois group $\mathrm{G}_{p}^{1}(k):=\operatorname{Gal}\left(\mathrm{F}_{p}^{1}(k) \mid k\right)$ of the maximal abelian unramified $p$-extension, that is the (first) Hilbert $p$-class field $\mathrm{F}_{p}^{1}(k)$, of $k$.

In the section $\S 1$, we defined the $p$-class tower group (briefly $p$-tower group) of $k$ as the Galois group $G=\mathrm{G}_{p}^{\infty}(k):=\operatorname{Gal}\left(\mathrm{F}_{p}^{\infty}(k) \mid k\right)$ of the maximal unramified pro- $p$ extension $\mathrm{F}_{p}^{\infty}(k)$ of $k$. The fixed field of the commutator subgroup $G^{\prime}$ (the minimal subgroup with abelian quotient) in $\mathrm{F}_{p}^{\infty}(k)$ is the maximal abelian unramified $p$-extension $\mathrm{F}_{p}^{1}(k)$ of $k$ with Galois group

$$
\operatorname{Gal}\left(\mathrm{F}_{p}^{1}(k) \mid k\right) \simeq \operatorname{Gal}\left(\mathrm{F}_{p}^{\infty}(k) \mid k\right) / \operatorname{Gal}\left(\mathrm{F}_{p}^{\infty}(k) \mid \mathrm{F}_{p}^{1}(k)\right)=G / G^{\prime} .
$$

Thus, the $p$-class group $\mathrm{Cl}_{p}(k)$ of $k$ is also isomorphic to the abelianization $G / G^{\prime}$ of the $p$-tower group $G$ of $k$. Consequently, the derived subgroup $G^{\prime}$ is a closed (and open) subgroup of finite index $\left(G: G^{\prime}\right)=p^{v}$ in the topological pro- $p$ group $G$, and there exist $v+1$ layers

$$
\left\{G^{\prime} \leq H \leq G \mid(G: H)=p^{n}\right\} \quad \text { for } \quad 0 \leq n \leq v
$$

of intermediate normal subgroups $H \unlhd G$ between $G$ and its commutator subgroup $G^{\prime}$. For each of them, we denote by $T_{G, H}: G \rightarrow H / H^{\prime}$ the Artin transfer homomorphism from $G$ to $H$ [2].

Definition 4.1. For each integer $0 \leq n \leq v$, the system

$$
\operatorname{Lyr}_{n}(G):=\left\{G^{\prime} \leq H \leq G \mid(G: H)=p^{n}\right\}
$$

of intermediate groups $G^{\prime} \leq H \leq G$ with index $(G: H)=p^{n}$ is called the $n t h$ layer of normal subgroups of $G$ with abelian quotients $G / H$. In particular, 
for $n=0, G$ forms the top layer $\operatorname{Lyr}_{0}(G)=\{G\}$, and for $n=v, G^{\prime}$ constitutes the bottom layer $\operatorname{Lyr}_{v}(K)=\left\{G^{\prime}\right\}$.

Since the abelianization $H^{\mathrm{ab}}=H / H^{\prime}$ forms the target of the Artin transfer homomorphism $T_{G, H}: G \rightarrow H / H^{\prime}$ from $G$ to $H$, we introduced a preliminary instance of the following terminology in [19, Dfn. 1.1, p. 403].

Definition 4.2. For each integer $0 \leq n \leq v$, the family

$$
\tau_{n}(G):=\left(H / H^{\prime}\right)_{H \in \operatorname{Lyr}_{n}(G)}
$$

of abelianizations is called the $n$th layer of the multi-layered transfer target type (TTT) of $G$,

$$
\tau(G):=\left[\tau_{0}(G) ; \ldots ; \tau_{v}(G)\right] .
$$

For each integer $0 \leq n \leq v$, the family

$$
\varkappa_{n}(G):=\left(\operatorname{ker}\left(T_{G, H}\right)\right)_{H \in \operatorname{Lyr}_{n}(G)}
$$

of transfer kernels is called the $n$th layer of the multi-layered transfer kernel type (TKT) of $G$,

$$
\varkappa(G):=\left[\varkappa_{0}(G) ; \ldots ; \varkappa_{v}(G)\right] .
$$

Definition 4.3. By the Artin pattern of $G$ we understand the pair

$$
\operatorname{AP}(G):=(\tau(G) ; \varkappa(G))
$$

consisting of the multi-layered TTT $\tau(G)$ and the multi-layered TKT $\varkappa(G)$ of $G$.

Remark 4.1. Suppose that $0<n<v$. If an ordering is defined for the elements of $\operatorname{Lyr}_{n}(G)$, then the same ordering is applied to the members of the layer $\tau_{n}(G)$ and the TTT layer is called ordered. Otherwise, the TTT layer is called unordered or accumulated, since equal components are collected in powers with formal exponents denoting iteration. The same considerations apply to the TKT.

Since it is increasingly difficult to compute the structure of the $p$-class groups $\mathrm{Cl}_{p}(L)$ of extension fields $L$ with $[L: k]=p^{n}$ in higher layers with $n \geq 2$, it is frequently sufficient to make use of information in the first layer only, that is the layer of subgroups with index $p$. Therefore, N. B oston, M. R. Bush and F. H a j ir [10] invented the following first order approximation of the TTT, and we add a supplementary notion for the TKT.

Definition 4.4. The restriction

$$
\begin{aligned}
\tau^{(1)}(G) & :=\left[\tau_{0}(G) ; \tau_{1}(G)\right], \text { resp., } \\
\varkappa^{(1)}(G) & :=\left[\varkappa_{0}(G) ; \varkappa_{1}(G)\right],
\end{aligned}
$$

of the TTT $\tau(G)$, resp., TKT $\varkappa(G)$, to the zeroth and first layer is called the index-p abelianization data (IPAD), resp., index-p obstruction data (IPOD), 
of $G$, since the kernel is an obstruction for the injectivity of the induced Artin transfer homomorphism $\tilde{T}_{G, H}: G / G^{\prime} \rightarrow H / H^{\prime}$, and thus also for an embedding of the $p$-class group of the base field $K$ into the $p$-class group of an extension $L$.

So, the complete TTT is an extension of the IPAD. However, there also exists another extension of the IPAD which is not covered by the TTT. It is constructed from the usual IPAD $\left[\tau_{0}(G) ; \tau_{1}(G)\right]$ of $G$, firstly, by observing that $\tau_{1}(G)=$ $\left(H / H^{\prime}\right)_{H \in \operatorname{Lyr}_{1}(G)}$ can be viewed as $\tau_{1}(G)=\left(\tau_{0}(H)\right)_{H \in \operatorname{Lyr}_{1}(G)}$ and, secondly, by extending each $\tau_{0}(H)$ to the IPAD $\left[\tau_{0}(H) ; \tau_{1}(H)\right]$ of $H$. The same ideas can be applied to the TKT and IPOD.

Definition 4.5. The family

$$
\begin{aligned}
\tau^{(2)}(G) & :=\left[\tau_{0}(G) ;\left(\left[\tau_{0}(H) ; \tau_{1}(H)\right]\right)_{H \in \operatorname{Lyr}_{1}(G)}\right], \text { resp. } \\
\varkappa^{(2)}(G) & :=\left[\varkappa_{0}(G) ;\left(\left[\varkappa_{0}(H) ; \varkappa_{1}(H)\right]\right)_{H \in \operatorname{Lyr}_{1}(G)}\right]
\end{aligned}
$$

is called the iterated IPAD, resp., IPOD, of second order of $G$.

\section{Relation rank of the $p$-class tower group}

There is a fatal misprint in both, the Russian original of the paper [28] of 1964 by I. R. S h a far evi c h, and in the english translation of 1966, where the Dirichlet unit rank $r$ is missing in formula $\left(18^{\prime}\right)$. Therefore, we prove the following correction.

Theorem 5.1 (Corrected version of Theorem 6 by I. R. S h a farevich [28]). Let $p$ be a prime number and denote by $\zeta$ a primitive pth root of unity. Let $k$ be a number field with signature $\left(r_{1}, r_{2}\right)$ and torsionfree Dirichlet unit rank $r=r_{1}+r_{2}-1$, and let $S$ be a finite set of non-archimedean or real archimedean places of $k$. Assume that no place in $S$ divides $p$.

Then the relation rank $d_{2}\left(G_{S}\right)=\operatorname{dim}_{\mathbb{F}_{p}} \mathrm{H}^{2}\left(G_{S}, \mathbb{F}_{p}\right)$ of the Galois group $G_{S}=\operatorname{Gal}\left(k_{S} \mid k\right)$ of the maximal pro-p extension $k_{S}$ of $k$ which is unramified outside of $S$ is bounded from above by

$$
d_{2}\left(G_{S}\right) \leq \begin{cases}d_{1}\left(G_{S}\right)+r & \text { if } S \neq \emptyset \text { or } \zeta \notin k, \\ d_{1}\left(G_{S}\right)+r+1 & \text { if } S=\emptyset \text { and } \zeta \in k,\end{cases}
$$

where $d_{1}\left(G_{S}\right)=\operatorname{dim}_{\mathbb{F}_{p}} \mathrm{H}^{1}\left(G_{S}, \mathbb{F}_{p}\right)$ denotes the generator rank of $G_{S}$. 
P r o of. It suffices to prove the corrected statement for the case $S=\emptyset$ and $\zeta \in k$.

Let $J$, resp., $U_{S}$, be the group of idèles, resp., the subgroup of $S$-unit idèles, of $k$, and put $\sigma(S)=\operatorname{dim}_{\mathbb{F}_{p}} V_{S} /\left(k^{\times}\right)^{p}$, where $V_{S}=U_{S} J^{p} \cap k^{\times}$. We start with the second part of [28, $\S 4$, Thm. 5, p. 137] which states that the relation rank of $G_{S}$ is bounded from above by $d_{2}\left(G_{S}\right) \leq \sigma(S)$ if $S=\emptyset$ and $\zeta \in k$.

According to [28, $\S 1$, Thm. 1, p. 130], the generator rank of $G_{S}$ is given by

where

$$
d_{1}\left(G_{S}\right)=t(S)+\lambda(S)+\sigma(S)-r-\delta,
$$

$$
\begin{gathered}
t(S)=\#\left\{v \in S \mid \zeta \in k_{v}\right\}, \quad \lambda(S)=\sum_{v \in S, v \mid p}\left[k_{v}: \mathbb{Q}_{v}\right], \\
\delta=0 \quad \text { if } \quad \zeta \notin k \quad \text { and } \quad \delta=1 \quad \text { if } \quad \zeta \in k .
\end{gathered}
$$

Our assumptions $S=\emptyset$ and $\zeta \in k$ imply that $t(S)=0, \lambda(S)=0$ and $\delta=1$.

Together, we obtain

$$
\begin{aligned}
d_{2}\left(G_{S}\right)-d_{1}\left(G_{S}\right) & \leq \sigma(S)-(t(S)+\lambda(S)+\sigma(S)-r-\delta) \\
& =-t(S)-\lambda(S)+r+\delta \\
& =-0-0+r+1 \\
& =r+1
\end{aligned}
$$

that is

$$
d_{2}\left(G_{S}\right) \leq d_{1}\left(G_{S}\right)+r+1 .
$$

ExAmple 5.1. The first examples of fields, where a violation of the Shafarevich Theorem in the misprinted version occurred, have been found by A. Azizi, A. Zekhnini and M. Taous [4: The torsionfree unit rank of any bicyclic biquadratic field $k=\mathbb{Q}(\sqrt{-1}, \sqrt{d})(d>1$ squarefree $)$ with signature $\left(r_{1}, r_{2}\right)=$ $(0,2)$ is $r=r_{1}+r_{2}-1=1$. The particular fields with radicand $d=p_{1} p_{2} q$, where $p_{1} \equiv 1(\bmod 8), p_{2} \equiv 5(\bmod 8)$ and $q \equiv 3(\bmod 4)$ are prime numbers such that $\left(\frac{p_{1}}{p_{2}}\right)=-1,\left(\frac{p_{1}}{q}\right)=-1$ and $\left(\frac{p_{2}}{q}\right)=-1$, have a 2-class group $\mathrm{Cl}_{2}(k)$ of type $(2,2,2)$, a 2 -class tower of length $\ell_{2}(k)=2$ [4, and one of the 2-tower groups $\langle 32,35\rangle,\langle 64,181\rangle,\langle 128,984\rangle$, etc. [22, Fig. 2, p. 752], which all have $p$-multiplicator rank $\mu=5$ [15]. Since the relation rank $d_{2}(G)=\operatorname{dim}_{\mathbb{F}_{p}} \mathrm{H}^{2}\left(G, \mathbb{F}_{p}\right)$ of a finite $p$-group $G$ is bounded from below by its $p$-multiplicator rank $\mu(G)$ [20, $\S 14$, p. 178], our corrected formula admits the conclusion that the relation rank $d_{2}(G) \geq \mu(G)=5$ of the 2 -tower groups $\langle 32,35\rangle,\langle 64,181\rangle,\langle 128,984\rangle$, etc. is exactly equal to $d_{1}(G)+r+1=3+1+1=5$, whereas the misprinted formula yields the contradiction $d_{2}(G) \leq d_{1}(G)+1=3+1=4$. In this case, the two inequalities $\mu(G) \leq d_{2}(G) \leq d_{1}(G)+r+1$ degenerate to consecutive equations $\mu(G)=d_{2}(G)=d_{1}(G)+r+1$.

Slightly differently from our definitions in [20, Dfn. 21.2, p. 187] and [21, Dfn. 6.1, p. 301], we now redefine the concept of a cover. 
Definition 5.1. Let $\mathfrak{G}$ be a finite metabelian p-group.

(1) By the cover $\operatorname{cov}(\mathfrak{G})$ of $\mathfrak{G}$ we understand the set of all (isomorphism classes of) finite $p$-groups $H$ whose second derived quotient $H / H^{\prime \prime}$ is isomorphic to $\mathfrak{G}$ :

$$
\operatorname{cov}(\mathfrak{G}):=\left\{H|| H \mid<\infty, H / H^{\prime \prime} \simeq \mathfrak{G}\right\} .
$$

(2) For any nonnegative integer $n \geq 0$, let the $n$-cover of $\mathfrak{G}$ be defined by

$$
\operatorname{cov}_{n}(\mathfrak{G}):=\left\{H \in \operatorname{cov}(\mathfrak{G}) \mid 0 \leq d_{2}(H)-d_{1}(H) \leq n\right\} .
$$

\section{Remark 5.1.}

(1) In contrast to our earlier definitions, the cover $\operatorname{cov}(\mathfrak{G})$ in the new sense cannot be empty, since it certainly contains (the isomorphism class of) the group $\mathfrak{G}$ itself as the unique metabelian element.

(2) By the Burnside basis theorem, the defining conditions $0 \leq d_{2}(H)-$ $d_{1}(H) \leq n$ for the $n$-cover of $\mathfrak{G}$ could also be replaced by $d_{1}(\mathfrak{G}) \leq d_{2}(H) \leq$ $d_{1}(\mathfrak{G})+n$, since $d_{1}(H)=d_{1}(\mathfrak{G})$.

Definition 5.2. Let $k$ be a number field with $p$-class rank $\rho$, torsionfree Dirichlet unit rank $r$, and the second $p$-class group $\mathfrak{G}=\mathrm{G}_{p}^{2}(k)$. By the Shafarevich cover $\operatorname{cov}_{k}(\mathfrak{G})$ of $\mathfrak{G}$ with respect to $k$ (or to a class of similar number fields) we understand the $n$-cover of $\mathfrak{G}$ with $n=r+1$ if $k$ contains a primitive $p$ th root of unity, and $n=r$ otherwise. The Shafarevich cover can also be defined directly by

$$
\operatorname{cov}_{k}(\mathfrak{G}):=\left\{H \in \operatorname{cov}(\mathfrak{G}) \mid \rho \leq d_{2}(H) \leq \rho+n\right\},
$$

since $d_{1}(\mathfrak{G})=d_{1}\left(\mathfrak{G} / \mathfrak{G}^{\prime}\right)=d_{1}\left(\mathrm{Cl}_{p}(k)\right)=\rho$.

EXAMPLE 5.2. Note first that the various $n$-covers of $\mathfrak{G}$ form a non-descending chain of sets,

$$
\operatorname{cov}_{0}(\mathfrak{G}) \subseteq \operatorname{cov}_{1}(\mathfrak{G}) \subseteq \cdots \subseteq \operatorname{cov}(\mathfrak{G})
$$

The cardinality of the cover can probably take any value. Even for closely related groups, such as parent-descendant pairs, the values may be very different.

(1) Generally, if $\# \operatorname{cov}(\mathfrak{G})=1$ but the Shafarevich cover of $\mathfrak{G}$ with respect to $k$ is empty, then $\mathfrak{G}$ cannot be the second $p$-class group $\mathrm{G}_{p}^{2}(k)$ of $k$.

(2) For instance, the cover of the 3 -group $\mathfrak{G}=\langle 243,4\rangle$ with

$$
\operatorname{IPAD} \tau^{(1)}=\left[1^{2} ;\left(\left(1^{3}\right)^{3}, 21\right)\right] \text { and } \operatorname{TKT} \varkappa_{1}=(4111)
$$

consists of $\mathfrak{G}$ alone. However, since $d_{2}(\mathfrak{G}) \geq \mathrm{MR}(\mathfrak{G})=3$ (p-multiplicator rank), the Shafarevich cover, $\operatorname{cov}_{k}(\mathfrak{G})=\operatorname{cov}_{0}(\mathfrak{G})$, with respect to complex quadratic fields $k$, having this IPAD and TKT, is empty, since $r=0, k$ with $\rho=2$ does not contain a primitive third root of unity, and thus $n=0$. 
(3) The 3-group $\mathfrak{G}=\langle 729,45\rangle$ is the unique immediate descendant of $\langle 243,4\rangle$ possessing a GI-automorphism. It shares the common

$$
\operatorname{IPAD} \tau^{(1)}=\left[1^{2} ;\left(\left(1^{3}\right)^{3}, 21\right)\right] \text { and } \operatorname{TKT} \varkappa_{1}=(4111)
$$

with its parent, and is infinitely capable with nuclear rank $\mathrm{NR}(\mathfrak{G})=2$. As mentioned in [21, Cor. 6.2, p. 301], its cover $\operatorname{cov}(\mathfrak{G})=\mathcal{T}(\mathfrak{G})$ coincides with its complete infinite descendant tree, and even the Shafarevich cover with respect to complex quadratic fields $k$,

where

$$
\operatorname{cov}_{k}(\mathfrak{G})=\operatorname{cov}_{0}(\mathfrak{G})=\left\{S_{j} \mid j \geq 0\right\},
$$

$$
S_{j}=\langle 243,4\rangle(-\# 1 ; 1-\# 2 ; 1)^{j}-\# 1 ; 1-\# 2 ; 2
$$

denotes a series of Schur $\sigma$-groups starting with order $3^{8}=6561$ for $j=0$ [21, $§ 6.2 .2$, pp. 299-304], is infinite, according to L. B artholdi and M. R. Bush [5]. The Shafarevich cover with respect to real quadratic fields $K, \operatorname{cov}_{K}(\mathfrak{G})=\operatorname{cov}_{1}(\mathfrak{G})$, is more extensive, containing the four groups $\langle 2187,270 \ldots 273\rangle$ [21, Fig. 1, p. 302] as the smallest additional elements.

\section{Constraints for higher $p$-class groups}

Several constraints restrict the possibilities for higher $p$-class groups $\mathrm{G}_{p}^{n}(k)=$ $\operatorname{Gal}\left(\mathrm{F}_{p}^{n}(k) \mid k\right)$ with $n \geq 2$ or $n=\infty$, of a given class of algebraic number fields $k$. It is not always true that the most restrictive constraints are most helpful for finding a certain group $\mathrm{G}_{p}^{n}(k)$. For instance, it is sometimes easier to determine the $p$-tower group $\mathrm{G}_{p}^{\infty}(K)$ of a real quadratic field $K$ with looser conditions, which admit lower group orders, than of a complex quadratic field $k$ with very tight constraints, which can only be satisfied by groups with huge orders.

Let us compile a list of constraints proceeding from the most general to more particular cases:

(1) the Shafarevich bound for the relation rank of the $p$-tower group $G:=\mathrm{G}_{p}^{\infty}(k)$,

$$
d_{1}(G) \leq d_{2}(G) \leq d_{1}(G)+n,
$$

with $n=r+1$ if $\zeta \in k$ and $n=r$ if $\zeta \notin k$,

(2) the cardinality $\# \operatorname{cov}_{k}(\mathfrak{G})$ of the Shafarevich cover of the second $p$-class group $\mathfrak{G}:=\mathrm{G}_{p}^{2}(k)$,

(3) the existence of a generator inverting (GI-)automorphism of any $\mathrm{G}_{p}^{n}(K)$, with $n \geq 2$ or $n=\infty$, acting as inversion map on the abelianization,

(4) selection rules for the class $\operatorname{cl}(\mathfrak{G})$, due to $p$-class number relations for odd primes $p \geq 3$,

(5) the capitulation number $\nu=\#\left\{i \mid \varkappa_{1}(i)=0\right\}$, that is the number of total transfer kernels. 
The first two items of this list concern arbitrary number fields $k$, whereas the last three conditions are mainly motivated by (real or complex) quadratic fields $K=\mathbb{Q}(\sqrt{d})$, in particular, the very last item only by complex quadratic fields with $d<0$, which enforce $\nu=0$ [12, [16.

\section{Capitulation type c.18, (0122)}

\subsection{The ground state of capitulation type c.18}

Assume that $K$ is an algebraic number field with 3-class group $\mathrm{Cl}_{3}(K)$ of type $(3,3) \hat{=} 1^{2}$. Let $L_{1}, \ldots, L_{4}$ be the four unramified cyclic cubic extensions of $K$, and suppose that the 1st order Artin pattern $\operatorname{AP}^{(1)}(K)$ of $K$ is given by the IPAD $\tau^{(1)}(K)=\left[1^{2} ; \mathbf{2}^{\mathbf{2}}, 1^{3}, 21,21\right]$ and the IPOD $\varkappa^{(1)}(K)=\left[G^{\prime} ; \mathbf{G}, H_{1}, H_{2}, H_{2}\right]$, i.e., $\tau_{1}(K)=((9,9),(3,3,3),(9,3),(9,3))$ are the type invariants of the 3 -class groups of the $L_{i}$ and $\varkappa_{1}(K)=(0122)$ is the 3 -capitulation type of $K$ in the $L_{i}$.

Proposition 7.1 (D. C. M a y e r, 2010).

(1) The second 3 -class group $\mathfrak{G}=\mathrm{G}_{3}^{2}(K)$ of $K$ is isomorphic to the metabelian 3 -group $\langle 729,49\rangle$ and thus its relation rank satisfies the inequality $d_{2}(\mathfrak{G}) \geq$ $d_{1}(\mathfrak{G})+2$.

(2) Consequently, if $K$ is a number field with torsionfree unit rank $r=1$ and does not contain the third roots of unity (in particular, if $K=\mathbb{Q}(\sqrt{d})$ is a real quadratic field), then the length of the 3 -class field tower of $K$ must be $\ell_{3}(K) \geq 3$.

Proof.

(1) First, we prove that $\mathfrak{G}$ must have coclass $\operatorname{cc}(\mathfrak{G})=2$. This can be done in two ways, either using $\tau_{1}(K)$ alone or using $\varkappa_{1}(K)$ alone.

- According to items 1) and 3) of [21, Thm. 3.2, p. 291], $\tau_{1}(\mathfrak{G})=\tau_{1}(K)$ must contain three components of type $1^{2}$ if $\operatorname{cc}(\mathfrak{G})=1$, and two components of type $1^{3}$ if $\operatorname{cc}(\mathfrak{G}) \geq 3$, whence $\operatorname{cc}(\mathfrak{G})=2$ is the only possibility for $\tau_{1}(\mathfrak{G})=\left(2^{2}, 1^{3},(21)^{2}\right)$ without any $1^{2}$ and with only one $1^{3}$.

- According to [17, Thm. 2.5, p. 479], $\varkappa_{1}(\mathfrak{G})=\varkappa_{1}(K)$ must contain three total kernels, designated by 0 , if $\operatorname{cc}(\mathfrak{G})=1$. Since all metabelian 3 -groups of coclass bigger than 2 are descendants of $\left\langle 3^{5}, 3\right\rangle$, and the TKT (2100) of this root contains a 2-cycle, the TKT $\varkappa_{1}(\mathfrak{G})$ must also contain a 2-cycle, if $\operatorname{cc}(\mathfrak{G}) \geq 3$, according to [11, Cor. 3.0.2, p. 772]. Therefore, the only possibility for $\varkappa_{1}(\mathfrak{G})=(0122)$ without a 2 -cycle and with only one 0 is $\operatorname{cc}(\mathfrak{G})=2$. 
Next, we show that $\mathfrak{G}$ must be a descendant of $\left\langle 3^{5}, 6\right\rangle$. According to item 2) of [21, Thm. 3.1, p. 290], the polarized component $2^{2}$ of order $3^{4}$ of $\tau_{1}(\mathfrak{G})=\tau_{1}(K)$ cannot occur for a sporadic 3 -group outside of coclass- 2 trees, whence $\mathfrak{G}$ must be a vertex of one of the three coclass- 2 trees with metabelian mainline. Their roots are $\left\langle 3^{5}, 3\right\rangle$, resp., $\left\langle 3^{5}, 6\right\rangle$, resp., $\left\langle 3^{5}, 8\right\rangle$. All descendants of these roots have three stable components of their TTT, $\left(\left(1^{3}\right)^{2}, 21\right)$, resp., $\left(1^{3},(21)^{2}\right)$, resp., $\left((21)^{3}\right)$, whence $\tau_{1}(\mathfrak{G})=\left(2^{2}, 1^{3},(21)^{2}\right)$ unambiguously leads to a descendant of $\left\langle 3^{5}, 6\right\rangle$, by item 2) of [21, Thm. 3.2, p. 291]. Furthermore, we have $d_{2}(\mathfrak{G}) \geq \operatorname{MR}(\mathfrak{G})=4=d_{1}(\mathfrak{G})+2$, since the $p$-multiplicator rank is a lower bound for the relation rank.

Finally, the Artin pattern $\operatorname{AP}(\mathfrak{G})=\left(\varkappa_{1}(\mathfrak{G}), \tau_{1}(\mathfrak{G})\right)$ provides a sort of coordinate system in which the coclass tree with root $\left\langle 3^{5}, 6\right\rangle$ is embedded, with horizontal axis $\varkappa_{1}(\mathfrak{G})$ and vertical axis $\tau_{1}(\mathfrak{G})$. The polarization $2^{2}$ of $\tau_{1}(\mathfrak{G})=\left(2^{2}, 1^{3},(21)^{2}\right)$ determines the nilpotency $\operatorname{class} \operatorname{cl}(\mathfrak{G})=4$ and thus also the order $|\mathfrak{G}|=3^{6}$, according to item 2) of [21, Thm. 3.2, p. 291], since the defect $k=1$ is only possible for type H.4 (2122). The polarization 0 of $\varkappa_{1}(\mathfrak{G})=(0122)$ with stable components (122) unambiguously identifies the mainline vertex of order $3^{6}$, which is $\left\langle 3^{6}, 49\right\rangle$, according to Fig. 1 .

(2) According to [28, Thm. 6, p. 140], the relation rank $d_{2}(G)$ of the 3 -class tower group $G:=\operatorname{Gal}\left(\mathrm{F}_{3}^{\infty}(K) \mid K\right)$ of a number field $K$ with unit rank $r=r_{1}+r_{2}-1=1$ and $\zeta_{3} \notin K$ must satisfy $d_{2}(G) \leq d_{1}(G)+r=2+1=3$, whence $G \not \mathfrak{G}$ and $\ell_{3}(K)=\operatorname{dl}(G) \geq 3$.

Suppose now that, under the assumptions preceding Proposition 7.1 . $G=\mathrm{G}_{3}^{\infty}(K)$ denotes the 3-class tower group of $K$ and we are additionally given the 2nd order IPAD of $K$,

$$
\tau^{(2)}(K)=\left[1^{2} ;\left(\mathbf{2}^{\mathbf{2}} ; \tau_{1}\left(L_{1}\right)\right),\left(1^{3} ; \tau_{1}\left(L_{2}\right)\right),\left(21 ; \tau_{1}\left(L_{3}\right)\right),\left(21 ; \tau_{1}\left(L_{4}\right)\right)\right],
$$

with fixed $\tau_{1}\left(L_{1}\right)=\left(\left(21^{2}\right)^{4}\right)$ and $\tau_{1}\left(L_{3}\right)=\left(21^{2},(21)^{3}\right)$.

Theorem 7.1 (D. C. Mayer, Aug. 2015). Among the non-metabelian candidates for the 3-tower group $G$ of $K$, the immediate descendants of step size 1 of $\langle 729,49\rangle$ are distinguished by the following criteria:

(1) $\tau_{1}\left(L_{2}\right)=\left(21^{2},\left(\mathbf{2 1}^{\mathbf{2}}\right)^{\mathbf{3}},\left(1^{2}\right)^{9}\right), \tau_{1}\left(L_{4}\right)=\left(21^{2},(\mathbf{2 1})^{\mathbf{3}}\right) \Longleftrightarrow G \simeq\langle 2187, \mathbf{2 8 4}\rangle$,

(2) $\tau_{1}\left(L_{2}\right)=\left(21^{2},\left(\mathbf{1}^{\mathbf{3}}\right)^{\mathbf{3}},\left(1^{2}\right)^{9}\right), \quad \tau_{1}\left(L_{4}\right)=\left(21^{2},(\mathbf{3 1})^{\mathbf{3}}\right) \Longleftrightarrow G \simeq\langle 2187, \mathbf{2 9 1}\rangle$.

In both cases, we have derived length $\operatorname{dl}(G)=3$ and nilpotency class $\operatorname{cl}(G)=5$.

Proof. We use the p-group generation algorithm by M. F. New man [24] and E. A. O'Brien [25], which is implemented in our licence of the computational algebra system MAGMA [8], [9], [15], to construct the descendant tree $\mathcal{T}(R)$ of the root $R=\langle 243,6\rangle$, which is restricted to the coclass tree $\mathcal{T}^{2}(R)$ 
in Fig. 1 by ignoring the bifurcation at the not coclass-settled vertex $\langle 729,49\rangle$. In Fig. [3, however, all periodic bifurcations [20, $\S 21.2]$ in the complete descendant tree are taken into consideration, but the tree is pruned from all TKTs different from c.18. In parallel computation with the recursive tree construction, the iterated IPAD of second order $\tau^{(2)}(V)$ is determined for each vertex $V$ and non-metabelian vertices $H$ are checked for their second derived quotient $H / H^{\prime \prime}$. The construction can be terminated at order $3^{11}$, because several components of the 2 nd order IPAD become stable and the remaining components reveal a deterministic growth: we have

$$
\begin{aligned}
\tau_{1}\left(L_{2}\right)=\left(*,\left(\mathbf{1}^{\mathbf{3}}\right)^{\mathbf{3}},\left(1^{2}\right)^{9}\right), \quad \tau_{1}\left(L_{3}\right)=\tau_{1}\left(L_{4}\right)= & \left(*,(\mathbf{2 1})^{\mathbf{3}}\right) \\
& \text { for vertices of coclass } \mathbf{2}
\end{aligned}
$$

and

$$
\begin{aligned}
\tau_{1}\left(L_{2}\right)=\left(*,\left(\mathbf{2 1}^{\mathbf{2}}\right)^{\mathbf{3}},\left(1^{2}\right)^{9}\right), \tau_{1}\left(L_{3}\right)=\tau_{1}\left(L_{4}\right)= & \left(*,(\mathbf{3 1})^{\mathbf{3}}\right) \\
& \text { for vertices of coclass } \mathbf{3} .
\end{aligned}
$$

Therefore, the vertices $\langle 2187,284\rangle$ and $\langle 2187,291\rangle$ are characterized uniquely by their iterated IPAD of second order, and the cover of their common parent is given by

$$
\operatorname{cov}(\langle 729,49\rangle)=\{\langle 729,49\rangle,\langle 2187,284\rangle,\langle 2187,291\rangle\}
$$

\subsection{Real quadratic fields of type c.18}

Proposition 7.2 (D. C. Mayer, Feb. 2010). In the range $0<d<10^{7}$ of fundamental discriminants d of real quadratic fields $K=\mathbb{Q}(\sqrt{d})$, there exist precisely 28 cases with 3-capitulation type $\varkappa_{1}(K)=(0122)$. They all share the common IPAD $\tau^{(1)}(K)=\left[1^{2} ;\left(\mathbf{2}^{2}, 1^{3},(21)^{2}\right)\right]$.

Proof. The results [18, Tbl. 6.5, p. 452], where the entry in column freq. should be 28 instead of 29 in the first row and 4 instead of 3 in the fourth row, were computed by means of the free number theoretic computer algebra system PARI/GP [26] using an implementation of our own principalization algorithm in a PARI script, as described in detail in [18, $\S 5$, pp. 446-450].

Theorem 7.2 (D. C. M a y e r, Aug. 2015).

(1) The $\mathbf{1 0}$ real quadratic fields $K=\mathbb{Q}(\sqrt{d})$ with the following discriminants $d$ (36\% of 28$)$,

$1030117, \quad 3259597, \quad 3928632, \quad 4593673, \quad 5327080$,

$5909813,7102277, \quad 7738629, \quad 7758589, \quad 9583736$,

have 3 -class tower group $G \simeq\left\langle 3^{7}, \mathbf{2 8 4}\right\rangle$ and 3 -tower length $\ell_{3}(K)=3$. 
(2) The 18 real quadratic fields $K=\mathbb{Q}(\sqrt{d})$ with the following discriminants $d$ (64\% of 28 ),

$534824, \quad 2661365, \quad 2733965, \quad 3194013, \quad 3268781$, $4006033, \quad 5180081, \quad 5250941, \quad 5489661, \quad 6115852$, $6290549, \quad 7712184, \quad 7857048, \quad 7943761, \quad 8243113$, $8747997, \quad 8899661, \quad 9907837$,

have 3 -class tower group $G \simeq\left\langle 3^{7}, \mathbf{2 9 1}\right\rangle$ and 3 -tower length $\ell_{3}(K)=3$.

Proof. Since all these real quadratic fields $K=\mathbb{Q}(\sqrt{d})$ have 3-capitulation type $\varkappa_{1}(K)=(0122)$ and 1 st IPAD $\tau^{(1)}(K)=\left[1^{2} ; \mathbf{2}^{2}, 1^{3},(21)^{2}\right]$, and the 10 fields in the first list have 2nd IPAD

$$
\begin{array}{ll}
\tau_{1}\left(L_{1}\right)=\left(\left(21^{2}\right)^{4}\right), & \tau_{1}\left(L_{3}\right)=\left(21^{2},(21)^{3}\right), \\
\tau_{1}\left(L_{2}\right)=\left(21^{2},\left(\mathbf{2 1}^{\mathbf{2}}\right)^{\mathbf{3}},\left(1^{2}\right)^{9}\right), & \tau_{1}\left(L_{4}\right)=\left(21^{2},(\mathbf{2 1})^{\mathbf{3}}\right),
\end{array}
$$

whereas the 18 fields in the second list have 2nd IPAD

$$
\begin{array}{ll}
\tau_{1}\left(L_{1}\right)=\left(\left(21^{2}\right)^{4}\right), & \tau_{1}\left(L_{3}\right)=\left(21^{2},(21)^{3}\right), \\
\tau_{1}\left(L_{2}\right)=\left(21^{2},\left(\mathbf{1}^{\mathbf{3}}\right)^{\mathbf{3}},\left(1^{2}\right)^{9}\right), & \tau_{1}\left(L_{4}\right)=\left(21^{2},(\mathbf{3 1})^{\mathbf{3}}\right),
\end{array}
$$

the claim is a consequence of Theorem 7.1 .

Remark 7.1. Unpublished results of M. R. B us h show that there are 4318 real quadratic fields with discriminants $0<d<10^{9}$ having the IPAD in Proposition 7.2. For the remaining 4290 cases outside of the range $0<d<10^{7}$, we cannot specify the 3 -tower group, since the computations would require exceeding amounts of CPU time. However, according to Proposition 7.1 and Theorem 7.1 , we know for sure that the length of the 3 -tower is certainly $\ell_{3}(K)=3$.

Figure 3 visualizes the groups in Theorems $7.1,7.3$ and 7.5 and their population in Theorems 7.2, 7.4 and 7.6.

\subsection{The 1st excited state of capitulation type c.18}

As before, $K$ is an algebraic number field with 3-class group $\mathrm{Cl}_{3}(K)$ of type $(3,3) \hat{=} 1^{2}$. Let $L_{1}, \ldots, L_{4}$ be the four unramified cyclic cubic extensions of $K$, and suppose that the 1 st order Artin pattern $\operatorname{AP}^{(1)}(K)$ of $K$ is given by the

$$
\operatorname{IPAD} \tau^{(1)}(K)=\left[1^{2} ; \mathbf{3}^{\mathbf{2}}, 1^{3}, 21,21\right]
$$

and the

$$
\operatorname{IPOD} \varkappa^{(1)}(K)=\left[G^{\prime} ; \mathbf{G}, H_{1}, H_{2}, H_{2}\right],
$$

i.e., $\tau_{1}(K)=((27,27),(3,3,3),(9,3),(9,3))$ are the type invariants of the 3 -class groups of the $L_{i}$, and $\varkappa_{1}(K)=(0122)$ is the 3 -capitulation type of $K$ in the $L_{i}$. 


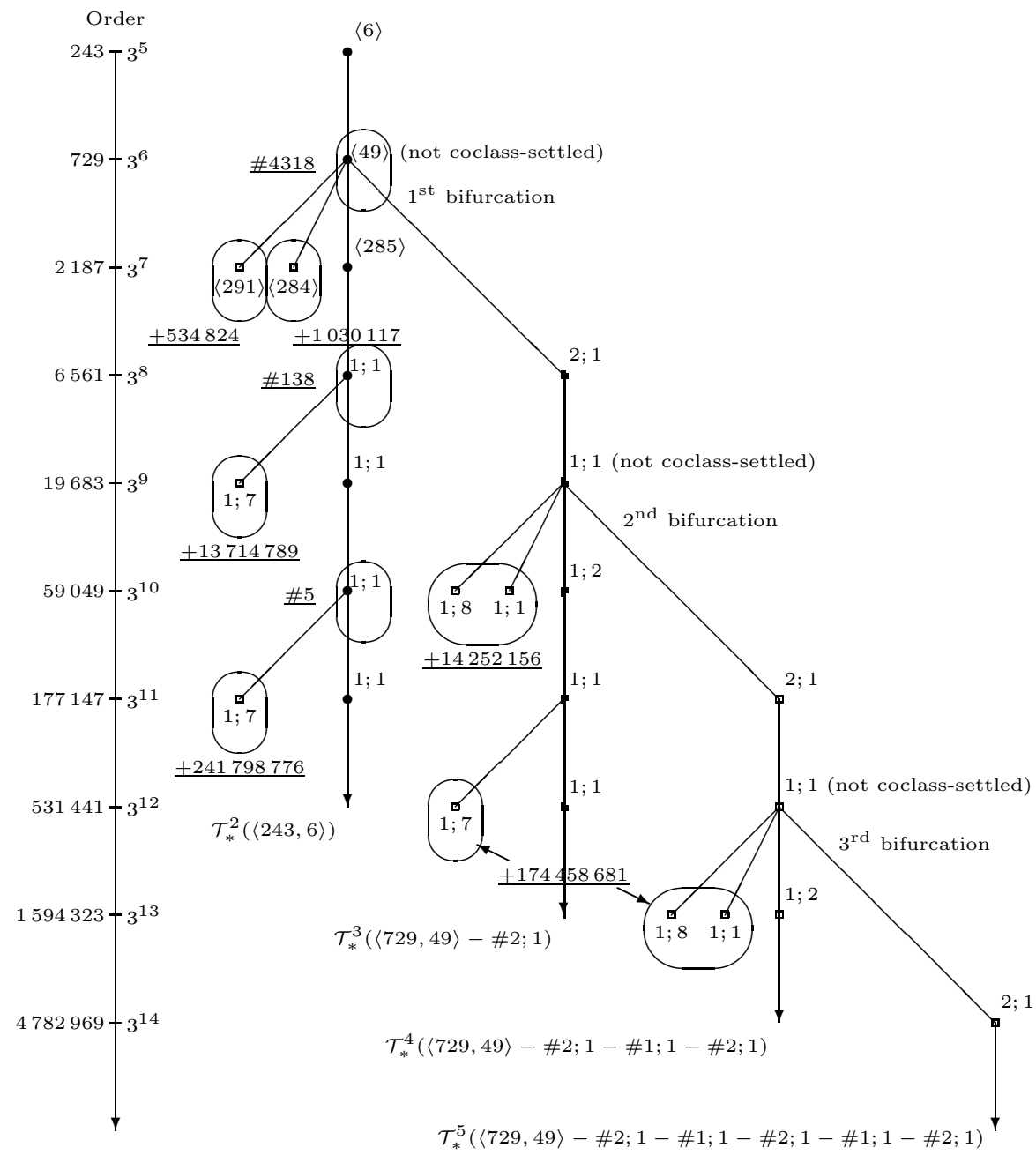

Figure 3. Non-metabelian 3-tower groups $G$ on the pruned tree $\mathcal{T}_{*}(\langle 243,6\rangle)$.

Proposition 7.3 (D. C. M a y e r, 2010).

(1) The second 3 -class group $\mathfrak{G}=\mathrm{G}_{3}^{2}(K)$ of $K$ is isomorphic to the metabelian 3 -group $\langle 2187,285\rangle-\# 1 ; 1$ and thus its relation rank satisfies the inequality $d_{2}(\mathfrak{G}) \geq d_{1}(\mathfrak{G})+2$.

(2) Consequently, if $K$ is a number field with torsionfree unit rank $r=1$ and does not contain the third roots of unity (in particular, if $K=\mathbb{Q}(\sqrt{d})$ is a real quadratic field), then the length of the 3 -class field tower of $K$ must be $\ell_{3}(K) \geq 3$. 
P r o of. A great deal of the proof is similar to the proof of Proposition 7.1

(1) First, we prove that $\mathfrak{G}$ must have coclass $\operatorname{cc}(\mathfrak{G})=2$. Next, we show that $\mathfrak{G}$ must be a descendant of $\left\langle 3^{5}, 6\right\rangle$, this time using the polarized component $3^{2}$ of order $3^{6}$ of $\tau_{1}(\mathfrak{G})$. Again, we have $d_{2}(\mathfrak{G}) \geq \operatorname{MR}(\mathfrak{G})=4=d_{1}(\mathfrak{G})+2$, since the $p$-multiplicator rank is a lower bound for the relation rank. Finally, the polarization $3^{2}$ of $\tau_{1}(\mathfrak{G})=\left(3^{2}, 1^{3},(21)^{2}\right)$ determines the nilpotency class $\operatorname{cl}(\mathfrak{G})=6$ and thus also the order $|\mathfrak{G}|=3^{8}$. The polarization 0 of $\varkappa_{1}(\mathfrak{G})=(0122)$ with stable components (122) unambiguously identifies the mainline vertex of order $3^{8}$, which is $\langle 2187,285\rangle-\# 1 ; 1$, according to Fig. 3 .

(2) As before, [28, Thm. 6, p. 140] implies that the relation rank $d_{2}(G)$ of the 3 -class tower group $G:=\operatorname{Gal}\left(\mathrm{F}_{3}^{\infty}(K) \mid K\right)$ of a number field $K$ with unit rank $r=r_{1}+r_{2}-1=1$ and $\zeta_{3} \notin K$ satisfies $d_{2}(G) \leq d_{1}(G)+r=2+1=3$, whence $G \not \mathfrak{G}$ and $\ell_{3}(K)=\operatorname{dl}(G) \geq 3$.

Suppose now that, under the assumptions preceding Prop.7.3. $G=\mathrm{G}_{3}^{\infty}(K)$ denotes the 3 -class tower group of $K$ and we are additionally given the 2 nd order IPAD of $K$,

$$
\tau^{(2)}(K)=\left[1^{2} ;\left(\mathbf{3}^{\mathbf{2}} ; \tau_{1}\left(L_{1}\right)\right),\left(1^{3} ; \tau_{1}\left(L_{2}\right)\right),\left(21 ; \tau_{1}\left(L_{3}\right)\right),\left(21 ; \tau_{1}\left(L_{4}\right)\right)\right],
$$

with fixed $\tau_{1}\left(L_{1}\right)=\left((321)^{4}\right)$.

Theorem 7.3 (D. C. M a y e r, Aug. 2015).

(1) $\tau_{1}\left(L_{2}\right)=\left(321,\left(\mathbf{1}^{\mathbf{3}}\right)^{\mathbf{3}},\left(1^{2}\right)^{9}\right)$ and $\tau_{1}\left(L_{i}\right)=\left(321,(\mathbf{2 1})^{\mathbf{3}}\right)$ for $i=3,4 \Longleftrightarrow$ $G \simeq\left\langle 3^{7}, 285\right\rangle-\# \mathbf{1} ; \mathbf{1}$, of order $|G|=3^{8}$, or

$G \simeq\left\langle 3^{7}, 285\right\rangle-\# \mathbf{1} ; \mathbf{1}-\# \mathbf{1} ; \mathbf{7}$, of order $|G|=3^{9}$,

(2) $\tau_{1}\left(L_{2}\right)=\left(321,\left(\mathbf{2 1}^{\mathbf{2}}\right)^{\mathbf{3}},\left(1^{2}\right)^{9}\right)$ and $\tau_{1}\left(L_{i}\right)=\left(321,(\mathbf{3 1})^{\mathbf{3}}\right)$ for $i=3,4 \Longleftrightarrow$

$G \simeq\left\langle 3^{6}, 49\right\rangle-\# \mathbf{2} ; \mathbf{1}-\# \mathbf{1} ; \mathbf{1}$, of order $|G|=3^{9}$, or

$G \simeq\left\langle 3^{6}, 49\right\rangle-\# \mathbf{2} ; \mathbf{1}-\# \mathbf{1} ; \mathbf{1}-\# \mathbf{1} ; \mathbf{1}$, of order $|G|=3^{10}$, or

$G \simeq\left\langle 3^{6}, 49\right\rangle-\# \mathbf{2} ; \mathbf{1}-\# \mathbf{1} ; \mathbf{1}-\# \mathbf{1} ; \mathbf{8}$, of order $|G|=3^{10}$.

P r o of. Similar as in the proof of Theorem 7.1 we construct the descendant tree $\mathcal{T}(R)$ of the root $R=\langle 243,6\rangle$, determine the iterated IPAD of second order $\tau^{(2)}(V)$ for each vertex $V$, and check the second derived quotient $H / H^{\prime \prime}$ of non-metabelian vertices $H$.

It turns out that the cover of the common metabelianization $\mathfrak{G}=\left\langle 3^{7}, 285\right\rangle-$ $\# 1 ; 1=G / G^{\prime \prime}$ of all candidates $G$ for the 3 -tower group is given by

$$
\begin{aligned}
\operatorname{cov}\left(\left\langle 3^{7}, 285\right\rangle-\# 1 ; 1\right)=\{ & \left\langle 3^{7}, 285\right\rangle-\# 1 ; 1,\left\langle 3^{7}, 285\right\rangle-\# 1 ; 1-\# 1 ; 7, \\
& \left\langle 3^{6}, 49\right\rangle-\# 2 ; 1-\# 1 ; 1,\left\langle 3^{6}, 49\right\rangle-\# 2 ; 1-\# 1 ; 1-\# 1 ; 1, \\
& \left.\left\langle 3^{6}, 49\right\rangle-\# 2 ; 1-\# 1 ; 1-\# 1 ; 8\right\} .
\end{aligned}
$$


The various vertices are not characterized uniquely by their iterated IPAD of second order. Rather they can be identified as batches of two resp., three vertices in the claimed manner.

Corollary 7.1 (D. C. M a y e r, Aug. 2015). If $K$ has torsionfree unit rank 1 and does not contain a primitive third root of unity, then

(1) $\tau_{1}\left(L_{2}\right)=\left(321,\left(\mathbf{1}^{\mathbf{3}}\right)^{\mathbf{3}},\left(1^{2}\right)^{9}\right)$ and $\tau_{1}\left(L_{i}\right)=\left(321,(\mathbf{2 1})^{\mathbf{3}}\right)$ for $i=3,4 \Longleftrightarrow$ $G \simeq\left\langle 3^{7}, 285\right\rangle-\# \mathbf{1} ; \mathbf{1}-\# \mathbf{1} ; \mathbf{7}$.

(2) $\tau_{1}\left(L_{2}\right)=\left(321,\left(\mathbf{2 1}^{\mathbf{2}}\right)^{\mathbf{3}},\left(1^{2}\right)^{9}\right)$ and $\tau_{1}\left(L_{i}\right)=\left(321,(\mathbf{3 1})^{\mathbf{3}}\right)$ for $i=3,4 \Longleftrightarrow$ $G \simeq\left\langle 3^{6}, 49\right\rangle-\# \mathbf{2} ; \mathbf{1}-\# \mathbf{1} ; \mathbf{1}-\# \mathbf{1} ; \mathbf{1}$ or $G \simeq\left\langle 3^{6}, 49\right\rangle-\# \mathbf{2} ; \mathbf{1}-\# \mathbf{1} ; \mathbf{1}-\# \mathbf{1} ; \mathbf{8}$.

All these groups have derived length $\mathrm{dl}(G)=3$.

P r o of. The two infinitely capable groups

$$
\left\langle 3^{7}, 285\right\rangle-\# \mathbf{1} ; \mathbf{1} \text { and }\left\langle 3^{6}, 49\right\rangle-\# \mathbf{2} ; \mathbf{1}-\# \mathbf{1} ; \mathbf{1}
$$

have $p$-multiplicator $\operatorname{rank} \operatorname{MR}(G)=4$ and thus relation $\operatorname{rank} d_{2}(G) \geq 4$, and consequently cannot satisfy the Shafarevich inequality

$$
d_{2}(G) \leq d_{1}(G)+r=2+1=3
$$

[28. Thm. 6, p. 140] for a field $K$ with unit rank $r=r_{1}+r_{2}-1=1$ and $\zeta_{3} \notin K$.

\subsection{Real quadratic fields of type c.18}

Proposition 7.4 (M. R. B u s h, Jul. 2015). In the range $0<d<10^{8}$ of fundamental discriminants d of real quadratic fields $K=\mathbb{Q}(\sqrt{d})$ there exist precisely 8 cases with the 1 st IPAD $\tau^{(1)}(K)=\left[1^{2} ; \mathbf{3}^{2}, 1^{3},(21)^{2}\right]$.

P r o o f. The results were communicated to us on July 11, 2015, by M. R. B u s h, who used PARI/GP [26] with similar techniques as described in our paper [18, § 5, pp. 446-450], and additionally double-checked with MAGMA [15].

Corollary 7.2 (D. C. Ma y e r, 2010). A quadratic field $K=\mathbb{Q}(\sqrt{d})$ with $\tau^{(1)}(K)=\left[1^{2} ; \mathbf{3}^{\mathbf{2}}, 1^{3},(21)^{2}\right]$ must be a real quadratic field with 3 -capitulation type $\varkappa_{1}(K)=(0122)$.

Pr o of. This is a consequence of item (1) in [18, Cor. 4.4.3, p. 442].

Theorem 7.4 (D. C. M a y e r, Aug. 2015).

(1) The 4 real quadratic fields $K=\mathbb{Q}(\sqrt{d})$ with the following discriminants $d$ (50\% of 8$)$,

$13714789,24037912,54683977,94272565$, have 3 -class tower group $G \simeq\left\langle 3^{7}, 285\right\rangle-\# \mathbf{1} ; \mathbf{1}-\# \mathbf{1} ; \mathbf{7}$. 
(2) The 4 real quadratic fields $K=\mathbb{Q}(\sqrt{d})$ with the following discriminants $d$ (50\% of 8$)$,

$14252156,46748181,67209369,78200897$,

have 3 -class tower group either

$G \simeq\left\langle 3^{6}, 49\right\rangle-\# \mathbf{2} ; \mathbf{1}-\# \mathbf{1} ; \mathbf{1}-\# \mathbf{1} ; \mathbf{1}$ or

$G \simeq\left\langle 3^{6}, 49\right\rangle-\# \mathbf{2} ; \mathbf{1}-\# \mathbf{1} ; \mathbf{1}-\# \mathbf{1} ; \mathbf{8}$.

In each case, the length of the 3 -class tower of $K$ is given by $\ell_{3}(K)=3$.

P r o of. Since all these real quadratic fields $K=\mathbb{Q}(\sqrt{d})$ have 3-capitulation type $\varkappa_{1}(K)=(0122)$, 1st IPAD $\tau^{(1)}(K)=\left[1^{2} ; \mathbf{3}^{\mathbf{2}}, 1^{3},(21)^{2}\right]$ and the suitable 2nd IPAD, the claim is a consequence of Corollary 7.1 .

Remark 7.2. Of course, the percentages given in Theorem 7.4 are unable to predict reliable tendencies for extensive statistical ensembles. The results of M. R. Bush show that there are 138 real quadratic fields with discriminants $0<d<10^{9}$ having the IPAD in Proposition 7.4. For the remaining 130 cases outside of the range $0<d<10^{8}$, we cannot specify the 3 -tower group, since the computation would require too much CPU time. However, according to Proposition 7.3 and Corollary [7.1] we can be sure that the length of the 3 -tower is exactly $\ell_{3}(K)=3$.

\subsection{The 2nd excited state of capitulation type c.18}

As before, $K$ is an algebraic number field with 3-class group $\mathrm{Cl}_{3}(K)$ of type $(3,3) \hat{=} 1^{2}$. Let $L_{1}, \ldots, L_{4}$ be the four unramified cyclic cubic extensions of $K$, and suppose that the $1^{\text {st }}$ order Artin pattern $\operatorname{AP}^{(1)}(K)$ of $K$ is given by the $\operatorname{IPAD} \tau^{(1)}(K)=\left[1^{2} ; \mathbf{4}^{\mathbf{2}}, 1^{3}, 21,21\right]$ and the IPOD $\varkappa^{(1)}(K)=\left[G^{\prime} ; \mathbf{G}, H_{1}, H_{2}, H_{2}\right]$, i.e., $\tau_{1}(K)=((81,81),(3,3,3),(9,3),(9,3))$ are the type invariants of the 3 -class groups of the $L_{i}$ and $\varkappa_{1}(K)=(0122)$ is the 3 -capitulation type of $K$ in the $L_{i}$.

Proposition 7.5 (D. C. M a y e r, 2010).

(1) The second 3 -class group $\mathfrak{G}=\mathrm{G}_{3}^{2}(K)$ of $K$ is isomorphic to the metabelian 3 -group $\langle 2187,285\rangle(-\# 1 ; 1)^{3}$ and thus its relation rank satisfies the inequality $d_{2}(\mathfrak{G}) \geq d_{1}(\mathfrak{G})+2$.

(2) Consequently, if $K$ is a number field with torsionfree unit rank $r=1$ and does not contain the third roots of unity (in particular, if $K=\mathbb{Q}(\sqrt{d})$ is a real quadratic field), then the length of the 3 -class field tower of $K$ must be $\ell_{3}(K) \geq 3$.

P r o o f. Again, a great deal of the proof is similar to the proof of Proposition 7.1,

(1) First, we prove that $\mathfrak{G}$ must have coclass $\operatorname{cc}(\mathfrak{G})=2$. Next, we show that $\mathfrak{G}$ must be a descendant of $\left\langle 3^{5}, 6\right\rangle$, this time using the polarized component $4^{2}$ 


\section{DANIEL C. MAYER}

of order $3^{8}$ of $\tau_{1}(\mathfrak{G})$. Again, we have

$$
d_{2}(\mathfrak{G}) \geq \operatorname{MR}(\mathfrak{G})=4=d_{1}(\mathfrak{G})+2,
$$

since the $p$-multiplicator rank is a lower bound for the relation rank. Finally, the polarization $4^{2}$ of $\tau_{1}(\mathfrak{G})=\left(4^{2}, 1^{3},(21)^{2}\right)$ determines the nilpotency class $\operatorname{cl}(\mathfrak{G})=8$ and thus also the order $|\mathfrak{G}|=3^{10}$. The polarization 0 of $\varkappa_{1}(\mathfrak{G})=(0122)$ with stable components (122) unambiguously identifies the mainline vertex of order $3^{10}$, which is $\langle 2187,285\rangle(-\# 1 ; 1)^{3}$, according to Fig. 3 .

(2) As before, [28, Thm. 6, p. 140] implies that the relation rank $d_{2}(G)$ of the 3-class tower group $G:=\operatorname{Gal}\left(\mathrm{F}_{3}^{\infty}(K) \mid K\right)$ of a number field $K$ with unit rank $r=r_{1}+r_{2}-1=1$ and $\zeta_{3} \notin K$ satisfies $d_{2}(G) \leq d_{1}(G)+r=2+1=3$, whence $G \not \mathfrak{G}$ and $\ell_{3}(K)=\operatorname{dl}(G) \geq 3$.

Suppose now that, under the assumptions preceding Proposition 7.5, $G=\mathrm{G}_{3}^{\infty}(K)$ denotes the 3-class tower group of $K$ and we are additionally given the 2 nd order IPAD of $K$,

$$
\tau^{(2)}(K)=\left[1^{2} ;\left(\mathbf{4}^{2} ; \tau_{1}\left(L_{1}\right)\right),\left(1^{3} ; \tau_{1}\left(L_{2}\right)\right),\left(21 ; \tau_{1}\left(L_{3}\right)\right),\left(21 ; \tau_{1}\left(L_{4}\right)\right)\right],
$$

with fixed $\tau_{1}\left(L_{1}\right)=\left((431)^{4}\right)$.

Theorem 7.5 (D. C. M a y e r, Sep. 2015).

(1) $\tau_{1}\left(L_{2}\right)=\left(431,\left(\mathbf{1}^{\mathbf{3}}\right)^{\mathbf{3}},\left(1^{2}\right)^{9}\right)$ and $\tau_{1}\left(L_{i}\right)=\left(431,(\mathbf{2 1})^{\mathbf{3}}\right)$ for $i=3,4 \Longleftrightarrow$

$G \simeq\left\langle 3^{7}, 285\right\rangle(-\# \mathbf{1} ; \mathbf{1})^{\mathbf{3}}$, of order $|G|=3^{10}$ or

$G \simeq\left\langle 3^{7}, 285\right\rangle(-\# \mathbf{1} ; \mathbf{1})^{\mathbf{3}}-\# \mathbf{1} ; \mathbf{7}$, of order $|G|=3^{11}$

(2) $\tau_{1}\left(L_{2}\right)=\left(431,\left(\mathbf{2 1}^{\mathbf{2}}\right)^{\mathbf{3}},\left(1^{2}\right)^{9}\right)$ and $\tau_{1}\left(L_{i}\right)=\left(431,(\mathbf{3 1})^{\mathbf{3}}\right)$ for $i=3,4 \Longleftrightarrow$

$G \simeq\left\langle 3^{6}, 49\right\rangle-\# \mathbf{2} ; \mathbf{1}-\# \mathbf{1} ; \mathbf{1}-\# \mathbf{1} ; \mathbf{2}-\# \mathbf{1} ; \mathbf{1}$, of order $|G|=3^{11}$ or

$G \simeq\left\langle 3^{6}, 49\right\rangle-\# \mathbf{2} ; \mathbf{1}-\# \mathbf{1} ; \mathbf{1}-\# \mathbf{1} ; \mathbf{2}-\# \mathbf{1} ; \mathbf{1}-\# \mathbf{1} ; \mathbf{7}$, of order $|G|=3^{12}$

or

$G \simeq\left\langle 3^{6}, 49\right\rangle(-\# \mathbf{2} ; \mathbf{1}-\# \mathbf{1} ; \mathbf{1})^{\mathbf{2}}$, of order $|G|=3^{12}$ or

$G \simeq\left\langle 3^{6}, 49\right\rangle(-\# \mathbf{2} ; \mathbf{1}-\# \mathbf{1} ; \mathbf{1})^{\mathbf{2}}-\# \mathbf{1} ; \mathbf{1}$, of order $|G|=3^{13}$ or

$G \simeq\left\langle 3^{6}, 49\right\rangle(-\# \mathbf{2} ; \mathbf{1}-\# \mathbf{1} ; \mathbf{1})^{\mathbf{2}}-\# \mathbf{1} ; \mathbf{8}$, of order $|G|=3^{13}$.

P r o of. Similar as in the proof of Theorem 7.1 we construct the descendant tree $\mathcal{T}(R)$ of the root $R=\langle 243,6\rangle$, determine the iterated IPAD of the second order $\tau^{(2)}(V)$ for each vertex $V$, and check the second derived quotient $H / H^{\prime \prime}$ of non-metabelian vertices $H$.

It turns out that the cover of the common metabelianization

$$
\mathfrak{G}=\left\langle 3^{7}, 285\right\rangle(-\# 1 ; 1)^{3}=G / G^{\prime \prime}
$$


of all candidates $G$ for the 3 -tower group is given by

$$
\begin{aligned}
\operatorname{cov} & \left(\left\langle 3^{7}, 285\right\rangle(-\# 1 ; 1)^{3}\right) \\
=\{ & \left\langle 3^{7}, 285\right\rangle(-\# 1 ; 1)^{3},\left\langle 3^{7}, 285\right\rangle(-\# 1 ; 1)^{3}-\# 1 ; 7,\left\langle 3^{6}, 49\right\rangle-\# 2 ; 1-\# 1 ; 1-\# 1 ; 2-\# 1 ; 1, \\
& \left\langle 3^{6}, 49\right\rangle-\# 2 ; 1-\# 1 ; 1-\# 1 ; 2-\# 1 ; 1-\# 1 ; 7,\left\langle 3^{6}, 49\right\rangle(-\# 2 ; 1-\# 1 ; 1)^{2}, \\
& \left.\left\langle 3^{6}, 49\right\rangle(-\# 2 ; 1-\# 1 ; 1)^{2}-\# 1 ; 1,\left\langle 3^{6}, 49\right\rangle(-\# 2 ; 1-\# 1 ; 1)^{2}-\# 1 ; 8\right\} .
\end{aligned}
$$

The various vertices are not characterized uniquely by their iterated IPAD of the second order. Rather they can be identified as batches of two resp., five vertices in the claimed manner.

Corollary 7.3 (D. C. M a y e r, Sep. 2015). If $K$ has torsionfree unit rank 1 and does not contain a primitive third root of unity, then

(1) $\tau_{1}\left(L_{2}\right)=\left(431,\left(\mathbf{1}^{\mathbf{3}}\right)^{\mathbf{3}},\left(1^{2}\right)^{9}\right)$ and $\tau_{1}\left(L_{i}\right)=\left(431,(\mathbf{2 1})^{\mathbf{3}}\right)$ for $i=3,4 \Longleftrightarrow$ $G \simeq\left\langle 3^{7}, 285\right\rangle(-\# \mathbf{1} ; \mathbf{1})^{\mathbf{3}}-\# \mathbf{1} ; \mathbf{7}$.

(2) $\tau_{1}\left(L_{2}\right)=\left(431,\left(\mathbf{2 1}^{\mathbf{2}}\right)^{\mathbf{3}},\left(1^{2}\right)^{9}\right)$ and $\tau_{1}\left(L_{i}\right)=\left(431,(\mathbf{3 1})^{\mathbf{3}}\right)$ for $i=3,4 \Longleftrightarrow$ $G \simeq\left\langle 3^{6}, 49\right\rangle-\# \mathbf{2} ; \mathbf{1}-\# \mathbf{1} ; \mathbf{1}-\# \mathbf{1} ; \mathbf{2}-\# \mathbf{1} ; \mathbf{1}-\# \mathbf{1} ; \mathbf{7}$ or

$G \simeq\left\langle 3^{6}, 49\right\rangle(-\# \mathbf{2} ; \mathbf{1}-\# \mathbf{1} ; \mathbf{1})^{\mathbf{2}}-\# \mathbf{1} ; \mathbf{1}$ or

$G \simeq\left\langle 3^{6}, 49\right\rangle(-\# \mathbf{2} ; \mathbf{1}-\# \mathbf{1} ; \mathbf{1})^{\mathbf{2}}-\# \mathbf{1} ; \mathbf{8}$.

All these groups have derived length $\operatorname{dl}(G)=3$.

P r o o f. The three groups $\left\langle 3^{7}, 285\right\rangle(-\# \mathbf{1} ; \mathbf{1})^{\mathbf{3}}$ and $\left\langle 3^{6}, 49\right\rangle-\# \mathbf{2} ; \mathbf{1}-\# \mathbf{1} ; \mathbf{1}-\# \mathbf{1}$; $\mathbf{2}-\# \mathbf{1} ; \mathbf{1}$ and $\left\langle 3^{6}, 49\right\rangle(-\# \mathbf{2} ; \mathbf{1}-\# \mathbf{1} ; \mathbf{1})^{\mathbf{2}}$, which are infinitely capable, have $p$-multiplicator $\operatorname{rank} \operatorname{MR}(G)=4$ and thus relation $\operatorname{rank} d_{2}(G) \geq 4$, and consequently cannot satisfy the Shafarevich inequality $d_{2}(G) \leq d_{1}(G)+r=2+1=3$ [28. Thm. 6, p. 140] for a field $K$ with unit rank $r=r_{1}+r_{2}-1=1$ and $\zeta_{3} \notin K$.

\subsection{Real quadratic fields of type c. $18 \uparrow^{2}$}

Proposition 7.6 (M. R. B u s h, Jul. 2015). In the range $0<d<10^{9}$ of fundamental discriminants d of real quadratic fields $K=\mathbb{Q}(\sqrt{d})$ there exist precisely 5 cases with 1 st IPAD $\tau^{(1)}(K)=\left[1^{2} ; \mathbf{4}^{\mathbf{2}}, 1^{3},(21)^{2}\right]$.

P r o o f. The results were communicated to us on July 11, 2015, by M. R. B u s h, who used PARI/GP [26] with similar techniques as described in our paper [18, $\S 5$, pp. 446-450], and additionally double-checked with MAGMA [15].

Corollary 7.4 (D. C. M a yer, 2010). A quadratic field $K=\mathbb{Q}(\sqrt{d})$ with $\tau^{(1)}(K)=\left[1^{2} ; \mathbf{4}^{2}, 1^{3},(21)^{2}\right]$ must be a real quadratic field with 3 -capitulation type $\varkappa_{1}(K)=(0122)$.

Pr o of. This is a consequence of item (1) in [18, Cor. 4.4.3, p. 442]. 
Theorem 7.6 (D. C. M a y e r, Sep. 2015).

(1) The single real quadratic fields $K=\mathbb{Q}(\sqrt{d})$ with discriminant $d=241798776(\mathbf{2 0} \%$ of 5$)$, has 3 -class tower group

$$
G \simeq\left\langle 3^{7}, 285\right\rangle(-\# \mathbf{1} ; \mathbf{1})^{\mathbf{3}}-\# \mathbf{1} ; \mathbf{7} .
$$

(2) The 4 real quadratic fields $K=\mathbb{Q}(\sqrt{d})$ with the following discriminants $d(\mathbf{8 0} \%$ of 5$)$,

$174458681,298160513,496930117,743138141$,

have 3 -class tower group either

$$
\begin{aligned}
& G \simeq\left\langle 3^{6}, 49\right\rangle-\# \mathbf{2} ; \mathbf{1}-\# \mathbf{1} ; \mathbf{1}-\# \mathbf{1} ; \mathbf{2}-\# \mathbf{1} ; \mathbf{1}-\# \mathbf{1} ; \mathbf{7} \text { or } \\
& G \simeq\left\langle 3^{6}, 49\right\rangle(-\# \mathbf{2} ; \mathbf{1}-\# \mathbf{1} ; \mathbf{1})^{\mathbf{2}}-\# \mathbf{1} ; \mathbf{1} \text { or } \\
& G \simeq\left\langle 3^{6}, 49\right\rangle(-\# \mathbf{2} ; \mathbf{1}-\# \mathbf{1} ; \mathbf{1})^{\mathbf{2}}-\# \mathbf{1} ; \mathbf{8} .
\end{aligned}
$$

In each case, the length of the 3 -class tower of $K$ is given by $\ell_{3}(K)=3$.

P r o of. Since all these real quadratic fields $K=\mathbb{Q}(\sqrt{d})$ have 3-capitulation type $\varkappa_{1}(K)=(0122)$, the 1 st IPAD $\tau^{(1)}(K)=\left[1^{2} ; \mathbf{4}^{\mathbf{2}}, 1^{3},(21)^{2}\right]$ and the suitable 2nd IPAD, the claim is a consequence of Corollary 7.3 .

\section{Capitulation type c.21, (2034)}

\subsection{The ground state of capitulation type c.21}

Assume that $K$ is an algebraic number field with 3-class group $\mathrm{Cl}_{3}(K)$ of type $(3,3) \hat{=} 1^{2}$. Let $L_{1}, \ldots, L_{4}$ be the four unramified cyclic cubic extensions of $K$, and suppose that the 1st order Artin pattern $\operatorname{AP}^{(1)}(K)$ of $K$ is given by the IPAD $\tau^{(1)}(K)=\left[1^{2} ; 21, \mathbf{2}^{2}, 21,21\right]$ and the IPOD $\varkappa^{(1)}(K)=\left[G^{\prime} ; H_{2}, \mathbf{G}, H_{3}, H_{4}\right]$, i.e., $\tau_{1}(K)=((9,3),(9,9),(9,3),(9,3))$ are the type invariants of the 3 -class groups of the $L_{i}$ and $\varkappa_{1}(K)=(2034)$ is the 3 -capitulation type of $K$ in the $L_{i}$.

Proposition 8.1 (D. C. M a y e r, 2010).

(1) The second 3 -class group $\mathfrak{G}=\mathrm{G}_{3}^{2}(K)$ of $K$ is isomorphic to the metabelian 3 -group $\langle 729,54\rangle$ and thus its relation rank satisfies the inequality $d_{2}(\mathfrak{G}) \geq$ $d_{1}(\mathfrak{G})+2$.

(2) Consequently, if $K$ is a number field with torsionfree unit rank $r=1$ and does not contain the third roots of unity (in particular, if $K=\mathbb{Q}(\sqrt{d})$ is a real quadratic field), then the length of the 3 -class field tower of $K$ must be $\ell_{3}(K) \geq 3$.

Proof.

(1) First, we prove that $\mathfrak{G}$ must have coclass $\operatorname{cc}(\mathfrak{G})=2$. This can be done in two ways, either using $\tau_{1}(K)$ alone or using $\varkappa_{1}(K)$ alone. 
- According to items 1) and 3) of [21, Thm. 3.2, p. 291], $\tau_{1}(\mathfrak{G})=\tau_{1}(K)$ must contain three components of type $1^{2}$ if $\operatorname{cc}(\mathfrak{G})=1$, and two components of type $1^{3}$ if $\operatorname{cc}(\mathfrak{G}) \geq 3$, whence $\operatorname{cc}(\mathfrak{G})=2$ is the only possibility for $\tau_{1}(\mathfrak{G})=\left(21,2^{2},(21)^{2}\right)$ without any $1^{2}$ and without any $1^{3}$.

- According to [17, Thm. 2.5, p. 479], $\varkappa_{1}(\mathfrak{G})=\varkappa_{1}(K)$ must contain three total kernels, designated by 0 , if $\operatorname{cc}(\mathfrak{G})=1$. Since all metabelian 3 -groups of coclass bigger than 2 are descendants of $\left\langle 3^{5}, 3\right\rangle$, and the TKT (2100) of this root contains a 2-cycle, the TKT $\varkappa_{1}(\mathfrak{G})$ must also contain a 2-cycle, if $\operatorname{cc}(\mathfrak{G}) \geq 3$, according to [11, Cor. 3.0.2, p. 772]. Therefore, the only possibility for $\varkappa_{1}(\mathfrak{G})=(2034)$ without a 2 -cycle and with only one 0 is $\operatorname{cc}(\mathfrak{G})=2$.

Next, we show that $\mathfrak{G}$ must be a descendant of $\left\langle 3^{5}, 8\right\rangle$. According to item 2) of [21, Thm. 3.1, p. 290], the polarized component $2^{2}$ of order $3^{4}$ of $\tau_{1}(\mathfrak{G})=\tau_{1}(K)$ cannot occur for a sporadic 3-group outside of coclass- 2 trees, whence $\mathfrak{G}$ must be a vertex of one of the three coclass- 2 trees with metabelian mainline. Their roots are $\left\langle 3^{5}, 3\right\rangle$, resp., $\left\langle 3^{5}, 6\right\rangle$, resp., $\left\langle 3^{5}, 8\right\rangle$. All descendants of these roots have three stable components of their TTT, $\left(\left(1^{3}\right)^{2}, 21\right)$, resp., $\left(1^{3},(21)^{2}\right)$, resp., $\left((21)^{3}\right)$, whence $\tau_{1}(\mathfrak{G})=\left(21,2^{2},(21)^{2}\right)$ unambiguously leads to a descendant of $\left\langle 3^{5}, 8\right\rangle$, by item 2) of [21, Thm. 3.2 , p. 291]. Furthermore, we have $d_{2}(\mathfrak{G}) \geq \operatorname{MR}(\mathfrak{G})=4=d_{1}(\mathfrak{G})+2$, since the $p$-multiplicator rank is a lower bound for the relation rank.

Finally, the Artin pattern $\operatorname{AP}(\mathfrak{G})=\left(\varkappa_{1}(\mathfrak{G}), \tau_{1}(\mathfrak{G})\right)$ provides a sort of coordinate system in which the coclass tree with root $\left\langle 3^{5}, 8\right\rangle$ is embedded, with horizontal axis $\varkappa_{1}(\mathfrak{G})$ and vertical axis $\tau_{1}(\mathfrak{G})$. The polarization $2^{2}$ of $\tau_{1}(\mathfrak{G})=\left(21,2^{2},(21)^{2}\right)$ determines the nilpotency class $\operatorname{cl}(\mathfrak{G})=4$ and thus also the order $|\mathfrak{G}|=3^{6}$, according to item 2) of [21, Thm. 3.2, p. 291], since the defect $k=1$ is only possible for type G.16 (2134). The polarization 0 of $\varkappa_{1}(\mathfrak{G})=(2034)$ with stable components (234) unambiguously identifies the mainline vertex of order $3^{6}$, which is $\left\langle 3^{6}, 54\right\rangle$, according to Fig. 2 ,

(2) According to [28, Thm. 6, p. 140], the relation rank $d_{2}(G)$ of the 3-class tower group $G:=\operatorname{Gal}\left(\mathrm{F}_{3}^{\infty}(K) \mid K\right)$ of a number field $K$ with torsionfree unit rank $r=r_{1}+r_{2}-1=1$ and $\zeta_{3} \notin K$ must satisfy $d_{2}(G) \leq d_{1}(G)+r=$

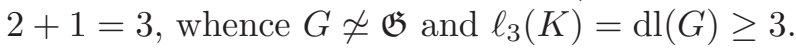

Suppose now that, under the assumptions preceding Proposition 8.1 . $G=\mathrm{G}_{3}^{\infty}(K)$ denotes the 3-class tower group of $K$ and we are additionally given the 2nd order IPAD of $K$,

$$
\tau^{(2)}(K)=\left[1^{2} ;\left(21 ; \tau_{1}\left(L_{1}\right)\right),\left(\mathbf{2}^{\mathbf{2}} ; \tau_{1}\left(L_{2}\right)\right),\left(21 ; \tau_{1}\left(L_{3}\right)\right),\left(21 ; \tau_{1}\left(L_{4}\right)\right)\right],
$$

with fixed $\tau_{1}\left(L_{1}\right)=\left(21^{2},(21)^{3}\right)$ and $\tau_{1}\left(L_{2}\right)=\left(\left(21^{2}\right)^{4}\right)$. 
Theorem 8.1 (D. C. M a y er, Aug. 2015). Among the non-metabelian candidates for the 3-tower group $G$ of $K$, the immediate descendants of step size 1 of $\langle 729,54\rangle$ are characterized by the following criteria:

(1) $\tau_{1}\left(L_{3}\right)=\left(21^{2},(21)^{3}\right), \tau_{1}\left(L_{4}\right)=\left(21^{2},(31)^{3}\right) \Longleftrightarrow G \simeq\langle 2187, \mathbf{3 0 7}\rangle$,

(2) $\tau_{1}\left(L_{3}\right)=\left(21^{2},(\mathbf{3 1})^{3}\right), \tau_{1}\left(L_{4}\right)=\left(21^{2},(21)^{3}\right) \Longleftrightarrow G \simeq\langle 2187,308\rangle$.

In both cases, we have derived length $\operatorname{dl}(G)=3$ and nilpotency class $\operatorname{cl}(G)=5$.

Remark 8.1. From the viewpoint of group theory, where a strict natural ordering can be imposed on the maximal subgroups of $G$ in terms of generators, the conditions of the two statements in Theorem 8.1 are distinct. From the viewpoint of number theory, however, no canonical ordering exists and the conditions are indistinguishable.

P r o of. In analogy to the proof of Theorem 7.1. we construct the descendant tree $\mathcal{T}(R)$ of the root $R=\langle 243,8\rangle$, which is restricted to the coclass tree $\mathcal{T}^{2}(R)$ in Fig. 2 by ignoring the bifurcation at the not coclass-settled vertex $\langle 729,54\rangle$. In Fig. [4, all periodic bifurcations [20, § 21.2] in the complete descendant tree are taken into consideration, but the tree is pruned from all TKTs different from c.21. In parallel computation with the recursive tree construction, the iterated IPAD of second order $\tau^{(2)}(V)$ is determined for each vertex $V$ and non-metabelian vertices $H$ are checked for their second derived quotient $H / H^{\prime \prime}$. The construction can be terminated at order $3^{11}$, because several components of the 2 nd order IPAD become stable and the remaining components reveal a deterministic growth: we have

$$
\tau_{1}\left(L_{1}\right)=\tau_{1}\left(L_{3}\right)=\tau_{1}\left(L_{4}\right)=\left(*,(\mathbf{2 1})^{\mathbf{3}}\right) \quad \text { for vertices of coclass } \mathbf{2}
$$

$$
\tau_{1}\left(L_{1}\right)=\tau_{1}\left(L_{3}\right)=\tau_{1}\left(L_{4}\right)=\left(*,(\mathbf{3 1})^{\mathbf{3}}\right) \quad \text { for vertices of coclass } \mathbf{3} .
$$

Therefore, the vertices $\langle 2187,307\rangle$ and $\langle 2187,308\rangle$ are characterized uniquely by their iterated IPAD of second order, and the cover of their common parent is given by

$$
\operatorname{cov}(\langle 729,54\rangle)=[\langle 729,54\rangle,\langle 2187,307\rangle,\langle 2187,308\rangle] .
$$

\subsection{Real quadratic fields of type c.21}

Proposition 8.2 (D. C. Mayer, Feb. 2010). In the range $0<d<10^{7}$ of fundamental discriminants d of real quadratic fields $K=\mathbb{Q}(\sqrt{d})$ there exist precisely 27 cases with 3 -capitulation type $\varkappa_{1}(K)=(2034)$. The 1st IPAD of $\mathbf{2 5}$ among them is $\tau^{(1)}(K)=[11 ; 21, \mathbf{2 2}, 21,21]$, the remaining 2 cases have $\tau^{(1)}(K)=[11 ; 21, \mathbf{3 3}, 21,21]$ (and will be considered in the section $\S 8.3$ ). 
P r o of. The results [18, Tbl. 6.7, p. 453] were computed by means of the free number theoretic computer algebra system PARI/GP [26] using an implementation of our own principalization algorithm in a PARI script, as described in detail in [18, $\S 5$, pp. 446-450].

Theorem 8.2 (D. C. M y er, August 2015). The 25 real quadratic fields $K=\mathbb{Q}(\sqrt{d})$ with the following discriminants $d$,

$\begin{array}{rrrrr}540365, & 945813, & 1202680, & 1695260, & 1958629, \\ 3018569, & 3236657, & 3687441, & 4441560, & 5512252, \\ 5571377, & 5701693, & 6027557, & 6049356, & 6054060, \\ 6274609, & 6366029, & 6501608, & 6773557, & 7573868, \\ 8243464, & 8251521, & 9054177, & 9162577, & 9967837,\end{array}$

have 3 -class tower group either $G \simeq\left\langle 3^{7}, \mathbf{3 0 7}\right\rangle$ or $G \simeq\left\langle 3^{7}, \mathbf{3 0 8}\right\rangle$.

In both cases, the length of the 3 -class tower of $K$ is given by $\ell_{3}(K)=3$.

P r o of. Since all these real quadratic fields $K=\mathbb{Q}(\sqrt{d})$ have 3-capitulation type $\varkappa_{1}(K)=(2034)$ and 1 st $\operatorname{IPAD} \tau^{(1)}(K)=\left[1^{2} ; 21, \mathbf{2}^{\mathbf{2}},(21)^{2}\right]$, and all 25 fields have unordered and indistinguishable 2nd IPAD

$$
\begin{array}{ll}
\tau_{1}\left(L_{1}\right)=\left(21^{2},(\mathbf{3 1})^{3}\right), & \tau_{1}\left(L_{3}\right)=\left(21^{2},(21)^{3}\right), \\
\tau_{1}\left(L_{2}\right)=\left(\left(21^{2}\right)^{4}\right), & \tau_{1}\left(L_{4}\right)=\left(21^{2},(21)^{3}\right),
\end{array}
$$

the claim is a consequence of Theorem 8.1 .

Remark 8.2. Unpublished results of M. R. B us h show that there are 4377 real quadratic fields with discriminants $0<d<10^{9}$ having the first IPAD in Proposition 8.2. For the remaining 4352 cases outside of the range $0<d<10^{7}$, we can only identify the 3 -tower group up to the two possibilities in Theorem 8.2 . However, according to Proposition 8.1 and Theorem 8.1, we know that the length of the 3 -tower is $\ell_{3}(K)=3$.

Figure 4 visualizes the groups in Theorems [8.1, 8.3 and 8.5] and their population in Theorems 8.2, 8.4 and 8.6 .

\subsection{The 1st excited state of capitulation type c. 21}

As before, $K$ is an algebraic number field with 3-class group $\mathrm{Cl}_{3}(K)$ of type $(3,3) \hat{=} 1^{2}$. Let $L_{1}, \ldots, L_{4}$ be the four unramified cyclic cubic extensions of $K$, and suppose that the 1st order Artin pattern $\operatorname{AP}^{(1)}(K)$ of $K$ is given by the $\operatorname{IPAD} \tau^{(1)}(K)=\left[1^{2} ; 21, \mathbf{3}^{2}, 21,21\right]$ and the IPOD $\varkappa^{(1)}(K)=\left[G^{\prime} ; H_{2}, \mathbf{G}, H_{3}, H_{4}\right]$, i.e., $\tau_{1}(K)=((9,3),(27,27),(9,3),(9,3))$ are the type invariants of the 3 -class groups of the $L_{i}$, and $\varkappa_{1}(K)=(2034)$ is the 3 -capitulation type of $K$ in the $L_{i}$. 


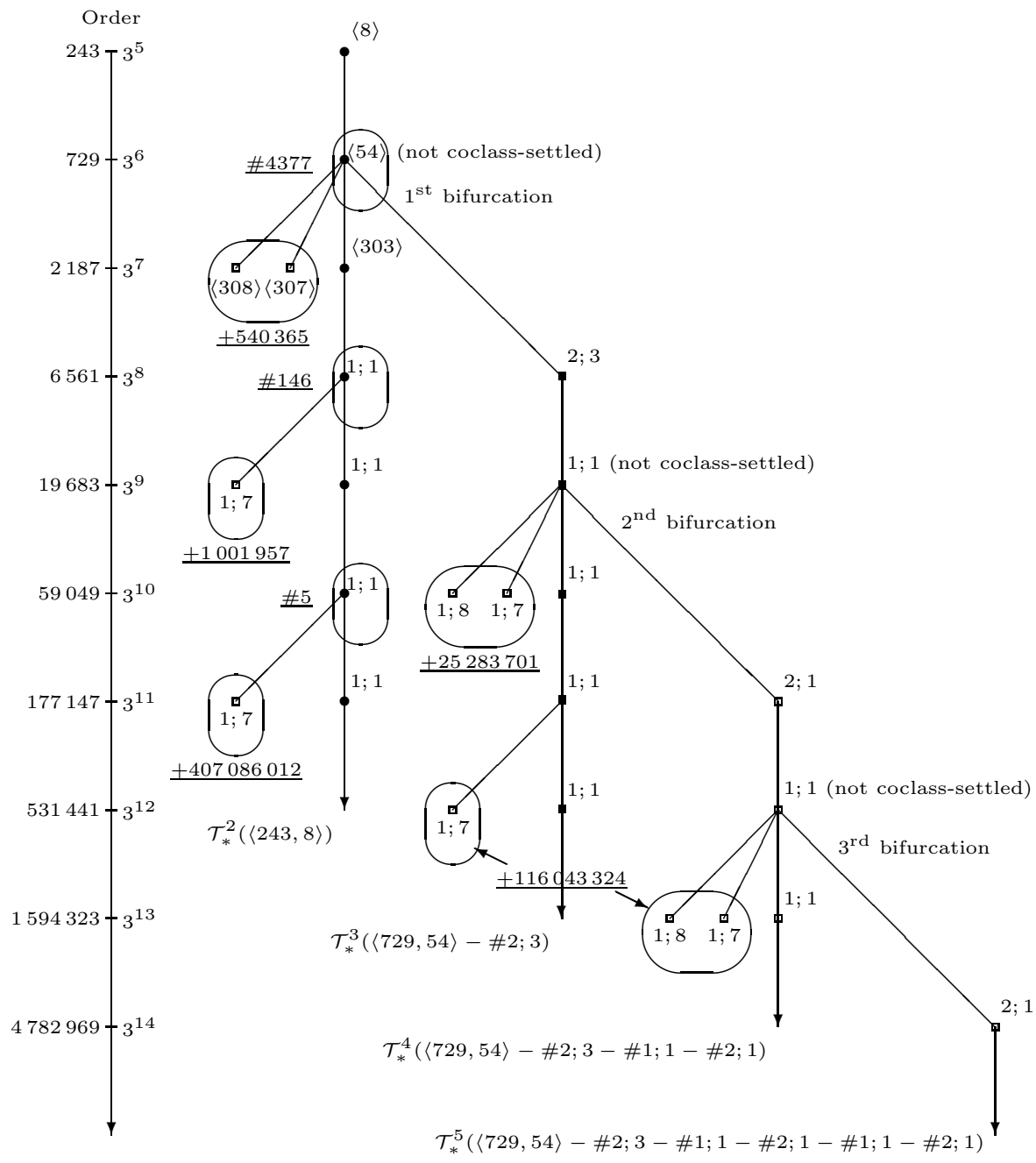

Figure 4. Non-metabelian 3-tower groups $G$ on the pruned tree $\mathcal{T}_{*}(\langle 243,8\rangle)$.

Proposition 8.3 (D. C. Ma y er, 2010).

(1) The second 3 -class group $\mathfrak{G}=\mathrm{G}_{3}^{2}(K)$ of $K$ is isomorphic to the metabelian 3 -group $\langle 2187,303\rangle-\# 1 ; 1$ and thus its relation rank satisfies the inequality $d_{2}(\mathfrak{G}) \geq d_{1}(\mathfrak{G})+2$.

(2) Consequently, if $K$ is a number field with torsionfree unit rank $r=1$ and does not contain the third roots of unity (in particular, if $K=\mathbb{Q}(\sqrt{d})$ is a real quadratic field), then the length of the 3-class field tower of $K$ must be $\ell_{3}(K) \geq 3$. 
P r o of. A great deal of the proof is similar to the proof of Proposition 8.1

(1) First, we prove that $\mathfrak{G}$ must have coclass $\operatorname{cc}(\mathfrak{G})=2$. Next, we show that $\mathfrak{G}$ must be a descendant of $\left\langle 3^{5}, 8\right\rangle$, this time using the polarized component $3^{2}$ of order $3^{6}$ of $\tau_{1}(\mathfrak{G})$. Again, we have $d_{2}(\mathfrak{G}) \geq \operatorname{MR}(\mathfrak{G})=4=d_{1}(\mathfrak{G})+2$, since the $p$-multiplicator rank is a lower bound for the relation rank. Finally, the polarization $3^{2}$ of $\tau_{1}(\mathfrak{G})=\left(21,3^{2},(21)^{2}\right)$ determines the nilpotency class $\operatorname{cl}(\mathfrak{G})=6$ and thus also the order $|\mathfrak{G}|=3^{8}$. The polarization 0 of $\varkappa_{1}(\mathfrak{G})=(2034)$ with stable components (234) unambiguously identifies the mainline vertex of order $3^{8}$, which is $\langle 2187,303\rangle-\# 1 ; 1$, according to Fig. 4

(2) As before, [28, Thm. 6, p. 140] implies that the relation rank $d_{2}(G)$ of the 3-class tower group $G:=\operatorname{Gal}\left(\mathrm{F}_{3}^{\infty}(K) \mid K\right)$ of a number field $K$ with unit rank $r=r_{1}+r_{2}-1=1$ and $\zeta_{3} \notin K$ satisfies $d_{2}(G) \leq d_{1}(G)+r=2+1=3$, whence $G \not \mathfrak{G}$ and $\ell_{3}(K)=\operatorname{dl}(G) \geq 3$.

Suppose now that, under the assumptions preceding Prop. 8.3, $G=\mathrm{G}_{3}^{\infty}(K)$ denotes the 3 -class tower group of $K$ and we are additionally given the 2 nd order IPAD of $K$,

$$
\tau^{(2)}(K)=\left[1^{2} ;\left(\left(21 ; \tau_{1}\left(L_{1}\right)\right),\left(\mathbf{3}^{2} ; \tau_{1}\left(L_{2}\right)\right),\left(21 ; \tau_{1}\left(L_{3}\right)\right),\left(21 ; \tau_{1}\left(L_{4}\right)\right)\right],\right.
$$

with fixed $\tau_{1}\left(L_{2}\right)=\left((321)^{4}\right)$.

Theorem 8.3 (D. C. M a y e r, Aug. 2015).

(1) $\tau_{1}\left(L_{i}\right)=\left(321,(\mathbf{2 1})^{\mathbf{3}}\right)$ for $i=1,3,4 \Longleftrightarrow$

$G \simeq\left\langle 3^{7}, 303\right\rangle-\# \mathbf{1} ; \mathbf{1}$, of order $|G|=3^{8}$ or

$G \simeq\left\langle 3^{7}, 303\right\rangle-\# \mathbf{1} ; \mathbf{1}-\# \mathbf{1} ; \mathbf{7}$, of order $|G|=3^{9}$

(2) $\tau_{1}\left(L_{i}\right)=\left(321,(\mathbf{3 1})^{\mathbf{3}}\right)$ for $i=1,3,4 \Longleftrightarrow$

$G \simeq\left\langle 3^{6}, 54\right\rangle-\# \mathbf{2} ; \mathbf{3}-\# \mathbf{1} ; \mathbf{1}$, of order $|G|=3^{9}$ or

$G \simeq\left\langle 3^{6}, 54\right\rangle-\# \mathbf{2} ; \mathbf{3}-\# \mathbf{1} ; \mathbf{1}-\# \mathbf{1} ; \mathbf{7}$, of order $|G|=3^{10}$ or

$G \simeq\left\langle 3^{6}, 54\right\rangle-\# \mathbf{2} ; \mathbf{3}-\# \mathbf{1} ; \mathbf{1}-\# \mathbf{1} ; \mathbf{8}$, of order $|G|=3^{10}$.

P r o of. Similar as in the proof of Theorem 8.1 we construct the descendant tree $\mathcal{T}(R)$ of the root $R=\langle 243,8\rangle$, determine the iterated IPAD of second order $\tau^{(2)}(V)$ for each vertex $V$, and check the second derived quotient $H / H^{\prime \prime}$ of non-metabelian vertices $H$.

It turns out that the cover of the common metabelianization

$$
\mathfrak{G}=\left\langle 3^{7}, 303\right\rangle-\# 1 ; 1=G / G^{\prime \prime}
$$

of all candidates $G$ for the 3 -tower group is given by $\operatorname{cov}\left(\left\langle 3^{7}, 303\right\rangle-\# 1 ; 1\right)=$

$$
\begin{aligned}
\left\{\left\langle 3^{7}, 303\right\rangle-\# 1 ; 1,\left\langle 3^{7}, 303\right\rangle-\# 1 ; 1-\# 1 ; 7,\left\langle 3^{6}, 54\right\rangle-\# 2 ; 3-\# 1 ; 1,\right. \\
\left.\left\langle 3^{6}, 54\right\rangle-\# 2 ; 3-\# 1 ; 1-\# 1 ; 7,\left\langle 3^{6}, 54\right\rangle-\# 2 ; 3-\# 1 ; 1-\# 1 ; 8\right\} .
\end{aligned}
$$


The various vertices are not characterized uniquely by their iterated IPAD of second order. Rather they can be identified as batches of two resp., three vertices in the claimed manner.

Corollary 8.1 (D. C. M a y e r, August 2015). If $K$ has torsionfree unit rank 1 and does not contain a primitive third root of unity, then

(1) $\tau_{1}\left(L_{i}\right)=\left(321,(\mathbf{2 1})^{3}\right)$ for $i=1,3,4 \Longleftrightarrow$ $G \simeq\left\langle 3^{7}, 303\right\rangle-\# \mathbf{1} ; \mathbf{1}-\# \mathbf{1} ; \mathbf{7}$.

(2) $\tau_{1}\left(L_{i}\right)=\left(321,(\mathbf{3 1})^{\mathbf{3}}\right)$ for $i=1,3,4 \Longleftrightarrow$ $G \simeq\left\langle 3^{6}, 54\right\rangle-\# \mathbf{2} ; \mathbf{3}-\# \mathbf{1} ; \mathbf{1}-\# \mathbf{1} ; \mathbf{7}$ or $G \simeq\left\langle 3^{6}, 54\right\rangle-\# \mathbf{2} ; \mathbf{3}-\# \mathbf{1} ; \mathbf{1}-\# \mathbf{1} ; \mathbf{8}$.

All these groups have derived length $\operatorname{dl}(G)=3$.

P r o of. The two infinitely capable groups

$$
\left\langle 3^{7}, 303\right\rangle-\# \mathbf{1} ; \mathbf{1} \text { and }\left\langle 3^{6}, 54\right\rangle-\# \mathbf{2} ; \mathbf{3}-\# \mathbf{1} ; \mathbf{1}
$$

have $p$-multiplicator $\operatorname{rank} \operatorname{MR}(G)=4$ and thus relation $\operatorname{rank} d_{2}(G) \geq 4$, and consequently cannot satisfy the Shafarevich inequality $d_{2}(G) \leq d_{1}(G)+r=$ $2+1=3$ [28, Thm. 6, p. 140] for a field $K$ with unit rank $r=r_{1}+r_{2}-1=1$ and $\zeta_{3} \notin K$.

\subsection{Real quadratic fields of type c.21个}

Proposition 8.4 (M. R. B u s h, Jul. 2015). In the range $0<d<10^{8}$ of fundamental discriminants d of real quadratic fields $K=\mathbb{Q}(\sqrt{d})$ there exist precisely 12 cases with 1 st IPAD $\tau^{(1)}(K)=\left[1^{2} ; 21, \mathbf{3}^{\mathbf{2}},(21)^{2}\right]$.

P r o o f. The results were communicated to us on July 11, 2015, by M. R. B u s h, who used PARI/GP [26] with similar techniques as described in our paper [18, $\S 5$, pp. 446-450], and additionally double-checked with MAGMA [15].

Corollary 8.2 (D. C. Ma y e r, 2010). A quadratic field $K=\mathbb{Q}(\sqrt{d})$ with $\tau^{(1)}(K)=\left[1^{2} ; 21, \mathbf{3}^{2},(21)^{2}\right]$ must be a real quadratic field with 3 -capitulation type $\varkappa_{1}(K)=(2034)$.

Proof. This is a consequence of item (1) in [18, Cor. 4.4.3, p. 442].

Theorem 8.4 (D. C. M a y er, August 2015).

(1) The 8 real quadratic fields $K=\mathbb{Q}(\sqrt{d})$ with the following discriminants $d$ (67\% of 12$)$,

$1001957, \quad 9923685, \quad 20633209, \quad 58650717$,

$63404792, \quad 72410413, \quad 84736636, \quad 92578472$,

have 3 -class tower group $G \simeq\left\langle 3^{7}, 303\right\rangle-\# \mathbf{1} ; \mathbf{1}-\# \mathbf{1} ; \mathbf{7}$. 
(2) The 4 real quadratic fields $K=\mathbb{Q}(\sqrt{d})$ with the following discriminants $d$ (33\% of 12$)$,

$25283701,36100840,42531528,81398865$,

have 3 -class tower group either

$$
\begin{aligned}
& G \simeq\left\langle 3^{6}, 54\right\rangle-\# \mathbf{2} ; \mathbf{3}-\# \mathbf{1} ; \mathbf{1}-\# \mathbf{1} ; \mathbf{7} \text { or } \\
& G \simeq\left\langle 3^{6}, 54\right\rangle-\# \mathbf{2} ; \mathbf{3}-\# \mathbf{1} ; \mathbf{1}-\# \mathbf{1} ; \mathbf{8} .
\end{aligned}
$$

In each case, the length of the 3 -class tower of $K$ is given by $\ell_{3}(K)=3$.

Pr o of. Since all these real quadratic fields $K=\mathbb{Q}(\sqrt{d})$ have 3-capitulation type $\varkappa_{1}(K)=(2034)$, 1st IPAD $\tau^{(1)}(K)=\left[1^{2} ; 21, \mathbf{3}^{\mathbf{2}},(21)^{2}\right]$ and suitable 2 nd IPAD, the claim is a consequence of Corollary 8.1 .

Remark 8.3. Of course, the percentages given in Theorem 8.4 are unable to predict reliable tendencies for extensive statistical ensembles. The results of M. R. Bush show that there are 146 real quadratic fields with discriminants $0<d<10^{9}$ having the IPAD in Proposition 8.4. For the remaining 134 cases outside of the range $0<d<10^{8}$, we cannot specify the 3 -tower group, since the computation would require too much CPU time. However, according to Proposition 8.3 and Corollary 8.1. we know that the length of the 3-tower is exactly $\ell_{3}(K)=3$.

\subsection{The 2nd excited state of capitulation type c.21}

As before, $K$ is an algebraic number field with 3-class group $\mathrm{Cl}_{3}(K)$ of type $(3,3) \hat{=} 1^{2}$. Let $L_{1}, \ldots, L_{4}$ be the four unramified cyclic cubic extensions of $K$, and suppose that the 1st order Artin pattern $\operatorname{AP}^{(1)}(K)$ of $K$ is given by the $\operatorname{IPAD} \tau^{(1)}(K)=\left[1^{2} ; 21,4^{2}, 21,21\right]$ and the IPOD $\varkappa^{(1)}(K)=\left[G^{\prime} ; H_{2}, \mathbf{G}, H_{3}, H_{4}\right]$, i.e., $\tau_{1}(K)=((9,3),(81,81),(9,3),(9,3))$ are the type invariants of the 3 -class groups of the $L_{i}$ and $\varkappa_{1}(K)=(2034)$ is the 3 -capitulation type of $K$ in the $L_{i}$.

Proposition 8.5 (D. C. M a y e r, 2010).

(1) The second 3 -class group $\mathfrak{G}=\mathrm{G}_{3}^{2}(K)$ of $K$ is isomorphic to the metabelian 3 -group $\langle 2187,303\rangle(-\# 1 ; 1)^{3}$ and thus its relation rank satisfies the inequality $d_{2}(\mathfrak{G}) \geq d_{1}(\mathfrak{G})+2$.

(2) Consequently, if $K$ is a number field with torsionfree unit rank $r=1$ and does not contain the third roots of unity (in particular, if $K=\mathbb{Q}(\sqrt{d})$ is a real quadratic field), then the length of the 3 -class field tower of $K$ must be $\ell_{3}(K) \geq 3$.

P r o o f. Again, a great deal of the proof is similar to the proof of Proposition 8.1 . 
(1) First, we prove that $\mathfrak{G}$ must have coclass $\operatorname{cc}(\mathfrak{G})=2$. Next, we show that $\mathfrak{G}$ must be a descendant of $\left\langle 3^{5}, 8\right\rangle$, this time using the polarized component $4^{2}$ of order $3^{8}$ of $\tau_{1}(\mathfrak{G})$. Again, we have

$$
d_{2}(\mathfrak{G}) \geq \operatorname{MR}(\mathfrak{G})=4=d_{1}(\mathfrak{G})+2,
$$

since the $p$-multiplicator rank is a lower bound for the relation rank. Finally, the polarization $4^{2}$ of $\tau_{1}(\mathfrak{G})=\left(21,4^{2},(21)^{2}\right)$ determines the nilpotency class $\operatorname{cl}(\mathfrak{G})=8$ and thus also the order $|\mathfrak{G}|=3^{10}$. The polarization 0 of $\varkappa_{1}(\mathfrak{G})=(2034)$ with stable components (234) unambiguously identifies the mainline vertex of order $3^{10}$, which is $\langle 2187,303\rangle(-\# 1 ; 1)^{3}$, according to Fig. 4 .

(2) As before, [28, Thm. 6, p. 140] implies that the relation rank $d_{2}(G)$ of the 3 -class tower group $G:=\operatorname{Gal}\left(\mathrm{F}_{3}^{\infty}(K) \mid K\right)$ of a number field $K$ with unit rank $r=r_{1}+r_{2}-1=1$ and $\zeta_{3} \notin K$ satisfies

$$
d_{2}(G) \leq d_{1}(G)+r=2+1=3,
$$

whence $G \not \mathfrak{G}$ and $\ell_{3}(K)=\operatorname{dl}(G) \geq 3$.

Suppose now that, under the assumptions preceding Prop. 8.5, $G=\mathrm{G}_{3}^{\infty}(K)$ denotes the 3-class tower group of $K$ and we are additionally given the 2nd order IPAD of $K$,

$$
\tau^{(2)}(K)=\left[1^{2} ;\left(\left(21 ; \tau_{1}\left(L_{1}\right)\right),\left(\mathbf{4}^{2} ; \tau_{1}\left(L_{2}\right)\right),\left(21 ; \tau_{1}\left(L_{3}\right)\right),\left(21 ; \tau_{1}\left(L_{4}\right)\right)\right],\right.
$$

with fixed $\tau_{1}\left(L_{2}\right)=\left((431)^{4}\right)$.

Theorem 8.5 (D. C. M a y e r, Sep. 2015).

(1) $\tau_{1}\left(L_{i}\right)=\left(431,(\mathbf{2 1})^{3}\right)$ for $i=1,3,4 \Longleftrightarrow$

$G \simeq\left\langle 3^{7}, 303\right\rangle(-\# \mathbf{1} ; \mathbf{1})^{\mathbf{3}}$, of order $|G|=3^{10}$ or

$G \simeq\left\langle 3^{7}, 303\right\rangle(-\# \mathbf{1} ; \mathbf{1})^{\mathbf{3}}-\# \mathbf{1} ; \mathbf{7}$, of order $|G|=3^{11}$

(2) $\tau_{1}\left(L_{i}\right)=\left(431,(\mathbf{3 1})^{3}\right)$ for $i=1,3,4 \Longleftrightarrow$

$G \simeq\left\langle 3^{6}, 54\right\rangle-\# \mathbf{2} ; \mathbf{3}(-\# \mathbf{1} ; \mathbf{1})^{\mathbf{3}}$, of order $|G|=3^{11}$ or

$G \simeq\left\langle 3^{6}, 54\right\rangle-\# \mathbf{2} ; \mathbf{3}(-\# \mathbf{1} ; \mathbf{1})^{\mathbf{3}}-\# \mathbf{1} ; \mathbf{7}$, of order $|G|=3^{12}$ or

$G \simeq\left\langle 3^{6}, 54\right\rangle-\# \mathbf{2} ; \mathbf{3}-\# \mathbf{1} ; \mathbf{1}-\# \mathbf{2} ; \mathbf{1}-\# \mathbf{1} ; \mathbf{1}$, of order $|G|=3^{12}$ or

$G \simeq\left\langle 3^{6}, 54\right\rangle-\# \mathbf{2} ; \mathbf{3}-\# \mathbf{1} ; \mathbf{1}-\# \mathbf{2} ; \mathbf{1}-\# \mathbf{1} ; \mathbf{1}-\# \mathbf{1} ; \mathbf{7}$, of order $|G|=3^{13}$ or

$G \simeq\left\langle 3^{6}, 54\right\rangle-\# \mathbf{2} ; \mathbf{3}-\# \mathbf{1} ; \mathbf{1}-\# \mathbf{2} ; \mathbf{1}-\# \mathbf{1} ; \mathbf{1}-\# \mathbf{1} ; \mathbf{8}$, of order $|G|=3^{13}$.

Proof. Similar as in the proof of Theorem 8.1 we construct the descendant tree $\mathcal{T}(R)$ of the root $R=\langle 243,8\rangle$, determine the iterated IPAD of second order $\tau^{(2)}(V)$ for each vertex $V$, and check the second derived quotient $H / H^{\prime \prime}$ of non-metabelian vertices $H$.

It turns out that the cover of the common metabelianization

$$
\mathfrak{G}=\left\langle 3^{7}, 303\right\rangle(-\# 1 ; 1)^{3}=G / G^{\prime \prime}
$$


of all candidates $G$ for the 3 -tower group is given by

$$
\begin{aligned}
& \operatorname{cov}\left(\left\langle 3^{7}, 303\right\rangle(-\# 1 ; 1)^{3}\right)= \\
& \left\{\left\langle 3^{7}, 303\right\rangle(-\# 1 ; 1)^{3},\left\langle 3^{7}, 303\right\rangle(-\# 1 ; 1)^{3}-\# 1 ; 7,\right. \\
& \quad\left\langle 3^{6}, 54\right\rangle-\# 2 ; 1-\# 1 ; 1-\# 1 ; 2-\# 1 ; 1, \\
& \left\langle 3^{6}, 54\right\rangle-\# 2 ; 1-\# 1 ; 1-\# 1 ; 2-\# 1 ; 1-\# 1 ; 7,\left\langle 3^{6}, 54\right\rangle(-\# 2 ; 1-\# 1 ; 1)^{2}, \\
& \left.\left\langle 3^{6}, 54\right\rangle(-\# 2 ; 1-\# 1 ; 1)^{2}-\# 1 ; 1,\left\langle 3^{6}, 54\right\rangle(-\# 2 ; 1-\# 1 ; 1)^{2}-\# 1 ; 8\right\} .
\end{aligned}
$$

The various vertices are not characterized uniquely by their iterated IPAD of second order. Rather they can be identified as batches of two resp., five vertices in the claimed manner.

Corollary 8.3 (D. C. M a y e r, Sep. 2015). If $K$ has torsionfree unit rank 1 and does not contain a primitive third root of unity, then

(1) $\tau_{1}\left(L_{i}\right)=\left(431,(\mathbf{2 1})^{3}\right)$ for $i=1,3,4 \Longleftrightarrow$ $G \simeq\left\langle 3^{7}, 303\right\rangle(-\# \mathbf{1} ; \mathbf{1})^{\mathbf{3}}-\# \mathbf{1} ; \mathbf{7}$.

(2) $\tau_{1}\left(L_{i}\right)=\left(431,(\mathbf{3 1})^{\mathbf{3}}\right)$ for $i=1,3,4 \Longleftrightarrow$ $G \simeq\left\langle 3^{6}, 54\right\rangle-\# \mathbf{2} ; \mathbf{3}(-\# \mathbf{1} ; \mathbf{1})^{\mathbf{3}}-\# \mathbf{1} ; \mathbf{7}$ or

$G \simeq\left\langle 3^{6}, 54\right\rangle-\# \mathbf{2} ; \mathbf{3}-\# \mathbf{1} ; \mathbf{1}-\# \mathbf{2} ; \mathbf{1}-\# \mathbf{1} ; \mathbf{1}-\# \mathbf{1} ; \mathbf{7}$ or

$G \simeq\left\langle 3^{6}, 54\right\rangle-\# \mathbf{2} ; \mathbf{3}-\# \mathbf{1} ; \mathbf{1}-\# \mathbf{2} ; \mathbf{1}-\# \mathbf{1} ; \mathbf{1}-\# \mathbf{1} ; \mathbf{8}$.

All these groups have derived length $\operatorname{dl}(G)=3$.

Proof. The three groups

$$
\begin{aligned}
& \left\langle 3^{6}, 54\right\rangle-\# \mathbf{2} ; \mathbf{3}-\# \mathbf{1} ; \mathbf{1}-\# \mathbf{2} ; \mathbf{1}-\# \mathbf{1} ; \mathbf{1}, \\
& \left\langle 3^{6}, 54\right\rangle-\# \mathbf{2} ; \mathbf{1}(-\# \mathbf{1} ; \mathbf{1})^{\mathbf{3}} \text { and } \\
& \left\langle 3^{7}, 303\right\rangle(-\# \mathbf{1} ; \mathbf{1})^{\mathbf{3}},
\end{aligned}
$$

which are infinitely capable, have $p$-multiplicator $\operatorname{rank} \operatorname{MR}(G)=4$ and thus relation rank $d_{2}(G) \geq 4$, and consequently cannot satisfy the Shafarevich inequality

$$
d_{2}(G) \leq d_{1}(G)+r=2+1=3
$$

[28, Thm. 6, p. 140] for a field $K$ with unit rank $r=r_{1}+r_{2}-1=1$ and $\zeta_{3} \notin K$.

\subsection{Real quadratic fields of type c. $21 \uparrow^{2}$}

Proposition 8.6 (M. R. B u s h, Jul. 2015). In the range $0<d<10^{9}$ of fundamental discriminants d of real quadratic fields $K=\mathbb{Q}(\sqrt{d})$ there exist precisely 5 cases with 1 st IPAD $\tau^{(1)}(K)=\left[1^{2} ; 21, \mathbf{4}^{\mathbf{2}},(21)^{2}\right]$. 
P r o o f. The results were communicated to us on July 11, 2015, by M. R. B u s h, who used PARI/GP [26] with similar techniques as described in our paper [18, § 5, pp. 446-450], and additionally double-checked with MAGMA [15].

Corollary 8.4 (D. C. Ma ye r, 2010). A quadratic field $K=\mathbb{Q}(\sqrt{d})$ with $\tau^{(1)}(K)=\left[1^{2} ; 21, \mathbf{4}^{2},(21)^{2}\right]$ must be a real quadratic field with 3 -capitulation type $\varkappa_{1}(K)=(2034)$.

Pr o of. This is a consequence of item (1) in [18, Cor. 4.4.3, p. 442].

Theorem 8.6 (D. C. M a y e r, Sep. 2015).

(1) The 4 real quadratic fields $K=\mathbb{Q}(\sqrt{d})$ with the following discriminants $d(\mathbf{8 0} \%$ of 5$)$,

$407086012,509164587,510908876,870946856$,

have 3 -class tower group $G \simeq\left\langle 3^{7}, 303\right\rangle(-\# \mathbf{1} ; \mathbf{1})^{\mathbf{3}}-\# \mathbf{1} ; \mathbf{7}$.

(2) The single real quadratic fields $K=\mathbb{Q}(\sqrt{d})$ with discriminant

$d=116043324$ (20\% of 5), has 3-class tower group either

$G \simeq\left\langle 3^{6}, 54\right\rangle-\# \mathbf{2} ; \mathbf{3}(-\# \mathbf{1} ; \mathbf{1})^{\mathbf{3}}-\# \mathbf{1} ; \mathbf{7}$ or

$G \simeq\left\langle 3^{6}, 54\right\rangle-\# \mathbf{2} ; \mathbf{3}-\# \mathbf{1} ; \mathbf{1}-\# \mathbf{2} ; \mathbf{1}-\# \mathbf{1} ; \mathbf{1}-\# \mathbf{1} ; \mathbf{7}$ or

$G \simeq\left\langle 3^{6}, 54\right\rangle-\# \mathbf{2} ; \mathbf{3}-\# \mathbf{1} ; \mathbf{1}-\# \mathbf{2} ; \mathbf{1}-\# \mathbf{1} ; \mathbf{1}-\# \mathbf{1} ; \mathbf{8}$.

In each case, the length of the 3 -class tower of $K$ is given by $\ell_{3}(K)=3$.

Proof. Since all these real quadratic fields $K=\mathbb{Q}(\sqrt{d})$ have 3-capitulation type $\varkappa_{1}(K)=(2034)$, 1st IPAD $\tau^{(1)}(K)=\left[1^{2} ; 21, \mathbf{4}^{2},(21)^{2}\right]$ and suitable 2nd IPAD, the claim is a consequence of Corollary 8.3 .

\section{Proof of the main result}

Combining several remarks and the results of two theorems, we are finally able to prove our initial Main Theorem 2.1.

P r o of. According to Remark 17.1. Remark 7.2 and Theorem 7.6, there exist exactly $4318+138+5=4461$ real quadratic fields $K=\mathbb{Q}(\sqrt{d})$ of type c. 18 , $\varkappa_{1}(K)=(0122)$, and according to Remark 8.2, Remark 8.3 and Theorem 8.6, there exist exactly $4377+146+5=4528$ real quadratic fields $K=\mathbb{Q}(\sqrt{d})$ of type c. $21, \varkappa_{1}(K)=(2034)$, in the range of fundamental discriminants $0<d<10^{9}$. They all have a 3 -class field tower of exact length $\ell_{3}(K)=3$. 


\section{When do we consider the $p$-class tower as "known"?}

As we have seen in this article, there are various pieces of information contributing to the knowledge of the $p$-class tower $\mathrm{F}_{p}^{\infty}(k)$ of a number field $k$ :

(1) the $p$-class tower group $G=\operatorname{Gal}\left(\mathrm{F}_{p}^{\infty}(k) \mid k\right)$ of $k$,

(2) the length $\ell_{p}(k)=\operatorname{dl}(G)$ of the $p$-class tower of $k$,

(3) iterated IPADs $\tau^{(n)}(G)$ and IPODs $\varkappa^{(n)}(G)$ of higher order $n \geq 2$,

(4) the second $p$-class group $\mathfrak{G}=\operatorname{Gal}\left(\mathrm{F}_{p}^{2}(k) \mid k\right) \simeq G / G^{\prime \prime}$ of $k$,

(5) the order $|\mathfrak{G}|$, nilpotency class $\operatorname{cl}(\mathfrak{G})$ and coclass $\operatorname{cc}(\mathfrak{G})$ of $\mathfrak{G}$,

(6) the annihilator ideal $\mathfrak{A}$ of the commutator subgroup $\mathfrak{G}^{\prime}$ of $\mathfrak{G}$,

(7) the $p$-capitulation kernels $\operatorname{ker}\left(j_{L \mid k}\right)$ of the unramified cyclic extensions $L \mid k$ of degree $p$, forming the first layer $\varkappa_{1}(k)$ of the $p$-capitulation type or TKT, of $k$,

(8) the $p$-class groups $\mathrm{Cl}_{p}(L)$ of the unramified cyclic extensions $L \mid k$ of degree $p$, forming the first layer $\tau_{1}(k)$ of the TTT of $k$,

(9) the $p$-class group $\mathrm{Cl}_{p}(k) \simeq G / G^{\prime}$ of $k$,

(10) the $p$-class rank $r_{p}(k)=\operatorname{dim}_{\mathbb{F}_{p}}\left(\mathbb{F}_{p} \otimes_{\mathbb{Z}_{p}} \mathrm{Cl}_{p}(k)\right)$ of $k$.

Figure 5 shows the logical implications between the pieces of information, which are valid generally, since they arise from mappings, such as $\mathrm{dl}(), \mathrm{cl}(), \mathrm{cc}()$, and so on.

However, in special cases, there exist further logical relations.

- If the $p$-class rank $r_{p}(k)$ of $k$ is sufficiently large, then the Golod-Shafarevich Theorem [13] of 1964 ensures an infinite $p$-class tower with length $\ell_{p}(k)=$ $\infty$, i.e., $(10) \rightarrow(2)$.

- Conversely, a finite $p$-class tower with length $\ell_{p}(k)<\infty$ enforces an upper bound on the $p$-class rank $r_{p}(k)$ of $k$, [13, i.e., $(2) \rightarrow(10)$.

- The Scholz-Taussky Theorem [27 of 1934 ensures a finite $p$-class tower with exact length $\ell_{p}(k)=2$, if the annihilator $\mathfrak{A}$ is the special ideal $\mathfrak{L}_{2}$, i.e., $(6) \rightarrow(2)$.

- For a group $\mathfrak{G}$ without defect of commutativity $(k(\mathfrak{G})=0)$, the knowledge of the nilpotency class $\operatorname{cl}(\mathfrak{G})=\alpha+1$ and the coclass $\operatorname{cc}(\mathfrak{G})=\beta$ of $\mathfrak{G}$ is equivalent with the knowledge of the annihilator ideal $\mathfrak{A}=\mathfrak{R}_{\alpha, \beta}$ and with the knowledge of the first layer $\tau_{1}(K)$ of the TTT of $\mathfrak{G}$, i.e., $(5) \leftrightarrow(6)$ and $(5) \leftrightarrow(8)$.

- For $p=3$ and $\mathrm{Cl}_{p}(k) \simeq(3,3)$, the TTT $\tau_{1}(k)$ occasionally determines the TKT $\varkappa_{1}(k)$, [18, Cor. 4.2.2, p. 436, and Cor. 4.3.2, p. 439], i.e., $(8) \rightarrow(7)$. 
(1) $G=\mathrm{G}_{p}^{\infty}(k)$

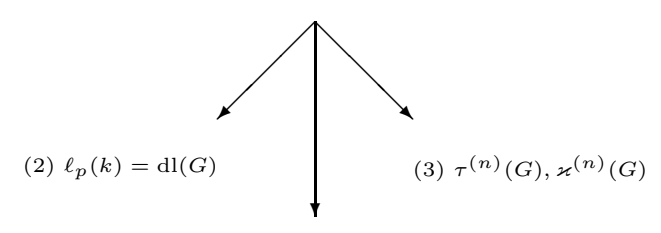

(4) $\mathfrak{G}=\mathrm{G}_{p}^{2}(k) \simeq G / G^{\prime \prime}$

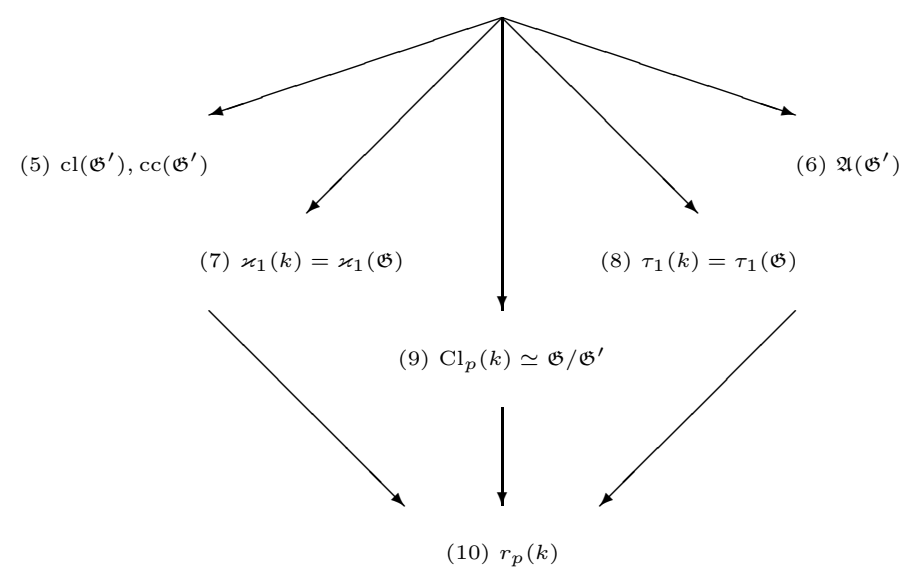

FiguRE 5. Pieces of information on a $p$-class tower $\mathrm{F}_{p}^{\infty}(k)$.

- For $p=5$ and $\mathrm{Cl}_{p}(k) \simeq(5,5)$, the TKT $\varkappa_{1}(k)$ occasionally determines the TTT $\tau_{1}(k)$ [19, Tbl. 3.11, p. 447, and the remark concerning the counter $\eta$ ], i.e., $(7) \rightarrow(8)$ or vice versa [19, Thm. 3.8 and Tbl. 3.3].

We are convinced that the $p$-class tower $\mathrm{F}_{p}^{\infty}(k)$ of a number field $k$ can only be considered as "known", if we are able to give a presentation of the $p$-class tower group $G=\operatorname{Gal}\left(\mathrm{F}_{p}^{\infty}(k) \mid k\right)$ and not merely to specify the $p$-tower length $\ell_{p}(k)$. This information permits to compute all data in Fig. 5 . Therefore, we made the initial Main Theorem 2.1 precise by stating and proving the detailed Theorems 7.2, 7.4, 7.6, 8.2, 8.4 and 8.6.

In view of future research, we point out that, although it is known [21, $\S 7$ ] that the complex quadratic field $k=\mathbb{Q}(\sqrt{d})$ with discriminant $d=4447704$ and 3 -class group of type $(3,3,3)$ has a 3 -class tower of length $\ell_{3}(k)=\infty$, according to the results of $\mathrm{H}$. $\mathrm{Koch}$ and $\mathrm{B}$. B. Venkov [14], there will remain a lot of work to do for establishing a pro-3 presentation of the 3-tower group $G=\operatorname{Gal}\left(\mathrm{F}_{3}^{\infty}(k) \mid k\right)$ of this mysterious field and determining the path from the root of the tree $\mathcal{T}(\langle 8,5\rangle)$ to its second 3-class group $\mathfrak{G}=\operatorname{Gal}\left(\mathrm{F}_{3}^{2}(k) \mid k\right) \simeq G / G^{\prime \prime}$. 
Acknowledgements. We gratefully acknowledge that our research is supported financially by the Austrian Science Fund (FWF): P 26008-N25.

We express our gratitude to M. F. New man (ANU, Canberra, ACT) for pointing out that the capable groups $G$ in Theorems 7.3 and 8.3 have $p$-multiplicator rank $\mu(G)=4$ and thus satisfy the inequality $d_{2}(G) \geq \mu(G)=$ $d_{1}(G)+2$ between their relation rank $d_{2}(G)$ and generator rank $d_{1}(G)=2$. This disqualifies them as candidates for $p$-class tower groups of real quadratic fields, according to the corrected Shafarevich Theorem 5.1.

Sincere thanks are given to M. R. B u sh (WLU, Lexington, VA) for making available brand-new numerical results on IPADs of real quadratic fields $K=\mathbb{Q}(\sqrt{d})$, and the distribution of discriminants $d<10^{9}$ over these IPADs, computed in July 2015.

We are indebted to N. B oston, M. R. Bush and F. Hajir for kindly making available an unpublished database containing numerical results of their paper [10] and a related paper on real quadratic fields, which is still in preparation.

A succinct version of this article has been presented at the 22nd Czech and Slovak International Conference on Number Theory in Liptovský Ján, Slovakia, on August 31, 2015.

\section{REFERENCES}

[1] ARTIN, E.: Beweis des allgemeinen Reziprozitätsgesetzes, Abh. Math. Sem. Univ. Hamburg 5 (1927), 353-363.

[2] ARTIN, E.: Idealklassen in Oberkörpern und allgemeines Reziprozitätsgesetz, Abh. Math. Sem. Univ. Hamburg 7 (1929), 46-51.

[3] ASCIONE, J. A.: On 3-groups of second maximal class. Ph.D. Thesis, Austral. National Univ., Canberra, 1979.

[4] AZIZI, A.-ZEKHNINI, A.-TAOUS, M.: Coclass of $\operatorname{Gal}\left(k_{2}^{(2)} \mid k\right)$ for some fields $k=\mathbb{Q}\left(\sqrt{p_{1} p_{2} q}, \sqrt{-1}\right)$ with 2-class groups of type $(2,2,2)$, J. Algebra Appl., 2015 (to appear).

[5] BARTHOLDI, L.- BUSH, M. R.: Maximal unramified 3-extensions of imaginary quadratic fields and $\mathrm{SL}_{2} \mathbb{Z}_{3}$, J. Number Theory 124 (2007), 159-166.

[6] BESCHE, H. U.-EICK, B.-O'BRIEN, E. A.: A millennium project: constructing small groups, Int. J. Algebra Comput. 12 (2002), 623-644.

[7] BESCHE, H. U.-EICK, B.-O'BRIEN, E. A.: The SmallGroups Library - a Library of Groups of Small Order, 2005, an accepted and refereed GAP package, available also in MAGMA.

[8] BOSMA, W.-CANNON, J.-PLAYOUST, C.: The Magma algebra system. I. The user language, J. Symbolic Comput. 24 (1997), 235-265. 


\section{DANIEL C. MAYER}

[9] BOSMA, W.-CANNON, J. J.-FIEKER, C.-STEELS, A. (EDS.): Handbook of Magma functions. Edition 2.21, Univ. of Sydney, Sydney, 2015.

[10] BOSTON, N.-BUSH, M. R.-HAJIR, F.: Heuristics for p-class towers of imaginary quadratic fields, Math. Annalen, 2015 (to appear); arXiv: 1111.4679v2 [math.NT] 10 Dec., 2014.

[11] BUSH, M. R.-MAYER, D. C.: 3-class field towers of exact length 3, J. Number Theory 147 (2015), 766-777.

[12] CHANG, S. M.-FOOTE, R.: Capitulation in class field extensions of type ( $p, p)$, Can. J. Math. 32 (1980), 1229-1243.

[13] GOLOD, E. S.-SHAFAREVICH, I. R.: On class field towers, Izv. Akad. Nauk SSSR, Ser. Mat. 28 (1964), no. 2, 261-272 (In Russian); English transl. in Amer. Math. Soc. Transl. (2) 48 (1965), 91-102.

[14] KOCH, H.-VENKOV, B. B.: Über den $p$-Klassenkörperturm eines imaginär-quadratischen Zahlkörpers, Astérisque 24-25 (1975), 57-67.

[15] The MAGMA Group, MAGMA Computational Algebra System, Version 2.21-8, Sydney, 2015, http://magma.maths.usyd.edu.au

[16] MAYER, D. C.: The second p-class group of a number field, Int. J. Number Theory 8 (2012), 471-505.

[17] MAYER, D. C.: Transfers of metabelian p-groups, Monatsh. Math. 166 (2012), 467-495.

[18] MAYER, D. C.: Principalization algorithm via class group structure, J. Théor. Nombres Bordeaux 26 (2014), 415-464.

[19] MAYER, D. C.: The distribution of second p-class groups on coclass graphs, J. Théor. Nombres Bordeaux 25 (2013), no. 2, 401-456; 27th Journées Arithmétiques, Faculty of Math. and Inform., University of Vilnius, Lithuania, 2011.

[20] MAYER, D. C.: Periodic bifurcations in descendant trees of finite p-groups, Adv. Pure Math. 5 (2015), 162-195.

[21] MAYER, D. C.: Index-p abelianization data of p-class tower groups, Adv. Pure Math. 5 (2015), 286-313; 29th Journées Arithmétiques, Univ. of Debrecen, Hungary, 2015.

[22] MAYER, D. C.: Periodic sequences of p-class tower groups, in: 1st Internat. Conference on Groups and Algebras-ICGA '15, Shanghai, China, 2015, J. Appl. Math. Phys. 3 (2015), 746-756.

[23] NEBELUNG, B.: Klassifikation metabelscher 3-Gruppen mit Faktorkommutatorgruppe vom Typ $(3,3)$ und Anwendung auf das Kapitulationsproblem. Inauguraldissertation, Universität zu Köln, 1989.

[24] NEWMAN, M. F.: Determination of groups of prime-power order, in: Proc. Miniconf. Canberra, Group Theory, 1975, Lecture Notes in Math., Vol. 573, Springer, Berlin, 1977, pp. $73-84$.

[25] O'BRIEN, E. A.: The p-group generation algorithm, J. Symbolic Comput. 9 (1990), 677-698.

[26] THE PARI GROUP, PARI/GP, Version 2.7.5, Bordeaux, 2015, http://pari.math.u-bordeaux.fr

[27] SCHOLZ, A.-TAUSSKY, O.: Die Hauptideale der kubischen Klassenkörper imaginär quadratischer Zahlkörper: ihre rechnerische Bestimmung und ihr Einfluß auf den Klassenkörperturm, J. Reine Angew. Math. 171 (1934), 19-41. 


\section{NEW NUMBER FIELDS WITH KNOWN $p$-CLASS TOWER}

[28] SHAFAREVICH, I. R.: Extensions with prescribed ramification points, Publ. Math., Inst. Hautes Études Sci. 18 (1964), 71-95, (In Russian); English transl. Amer. Math. Soc. Transl., II. Ser., 59 (1966), 128-149.

Received November 2, 2015

Naglergasse 53

AT-8010 Graz

AUSTRIA

E-mail: algebraic.number.theory@algebra.at 